\title{
Density Estimation of Small Mammals: Comparison of Techniques Utilizing Removal Trapping ${ }^{1}$
}

\author{
Donald W. KAUFMAN, John B. GENTRY, Glennis A. KAUFMAN, \\ Michael H. SMITH \& James G. WIENER
}

\begin{abstract}
Kaufman D. W., Gentry J. B., Kaufman G. A., Smith M. H. \& Wiener J. G., 1978: Density estimation of small mammals: comparison of techniques utilizing removal trapping. Acta theriol., 23, 7: 147-171 [With 10 Tables].

Removal trapping was conducted on $12 \times 12$ grids with assessment lines to test the relative effectiveness of the Standard Minimum, Inner Square and Assessment Line methods for small mammal density estimation. Twelve study sites were trapped in desert, chaparral and woodland habitats in California and Nevada to provide a wide array of species and densities for the comparison of estimation techniques. The procedure used was to trap each grid for 10 days followed by 4 days of trapping on the associated assessment lines.

Field sites were quite variable with a range of $12-167$ rodents per grid representing $2-10$ species. Density estimates ranged from $3.7-51 . \overline{5}$ animals/ha for the Standard Minimum method, 4.0-36.9 animals/ha for the Inner Square method and 0.7-114.4 animals/ha for the Assessment Line method. All methods provide relative density estimates for within method comparisons but their usefulness for estimating absolute density is limited. The Assessment Line technique probably yields the best absolute density estimates $\left(D_{A L}\right)$ when sufficient numbers of assessment lines are trapped; however, under most conditions a single $12 \times 12$ grid with 8 assessment lines is not sufficient. To obtain reliable $D_{A L}$ values, captures on assessment lines need to be combined for two or more grids from the same habitat to delineate the area of effect around a grid. Problems associated with each of the methods are discussed in detail.

Savannah River Ecol. Lab., SROO, Bldg. 772-G, Aiken, South Carolina 29801 (JBG, JGW); Dept. Biol. Sci., State Univ. New York, Binghamton, New York 13901 (DWK, GAK); Dept. Zool. \& Inst. Ecol., Univ. Georgia, Athens, Georgia 30601 (MHS)].
\end{abstract}

\section{INTRODUCTION}

A major objective of mammalian ecologists in recent years has been to develop a standard method of estimating small mammal density. In the search for such a method, several techniques have been suggested and tested (see review by S mith at al., 1975); however, the usefulness of any one method over the others has not been established. The major problem with analysis of the various density

1 This study was carried out under contract AT (38-1) - 310 between the U.S. Energy Research \& Development Administration and the University of Georgia. 
estimation techniques is that the true density in the area being studied is not known and the accuracy of the method relative to the true density cannot be ascertained. It is possible, though, to use the mathematical analyses for several removal trapping techniques with data from a single grid and compare the density values derived from the different estimators. Three techniques that can be analyzed relative to each other since they utilize the same basic trapping grid are the Standard Minimum, Inner Square and Assessment Line methods.

Our primary purpose in this paper is to compare the relative effectiveness of the three methods for the determination of absolute density of small mammals. Our analysis of these estimators utilizes data from 12 grids located in a variety of habitats in the southwestern United States which represent a wide array of densities and species of small mammals.

\section{METHODS}

Small mammals were sampled at each of six field sites in southern California and six sites in southern Nevada using $12 \times 12$ grids. Eight assessment lines were utilized with each grid (see Fig. 1D in Smith et al., 1975). The assessment lines extended $352.5 \mathrm{~m}$ from the center of the grid and consisted of 24 trap stations in California but were shortened to $247.5 \mathrm{~m}$ and 17 stations in Nevada. The interstation interval was $15 \mathrm{~m}$ for both grids and assessment lines. One Museum Special and one Victor mouse trap or one Museum Special mouse trap and one Victor rat trap were placed at each station. Traps were baited with peanut butter and checked daily. All grids were trapped for 10 consecutive days except Boulder and Joshua II which were trapped for 13 and 12 days, respectively. On day 11 (14 for Boulder and 13 for Joshua II) traps were removed from the grids and placed on assessment line stations. Traps were checked for four days on the assessment lines.

Sites trapped in California were located in desert habitats (Barrel, Boulder and Ocotillo near Ocotillo), chaparral (Chaparral I near Alpine and Chaparral II near El Cajon) and woodland (Juniper near Jacumba). Desert sites trapped in Nevada included Creosote I and II on the U.S. Energy Research \& Development Administration's Test Site near Mercury and Creosote III, Joshua I and II near the junction of Nevada Highway 52 and U. S. Highway 95 north of Las Vegas. One grid (Piñon) was trapped in the woodland located at the base of the Charleston Mountains near Lee Canyon. California sites were trapped during June and early July, 1970 whereas Nevada sites were trapped in late July and early August, 1970.

\section{MATHEMATICAL PROCEDURES FOR DENSITY ESTIMATION}

The basic equation for estimation of density $(D)$ is

$$
D=\frac{N}{A}
$$

where $N$ is the number of organisms and $A$ is the area from which $N$ is removed. For small mammals trapped on a grid, the generalized equation for calculating the area trapped $(A)$ is

$$
\mathrm{A}=\mathrm{W}_{G}^{2}+4 \mathrm{~W}_{G} \mathrm{~W}_{B}+\Pi \mathrm{W}_{B}^{2}
$$


where $W_{G}$ is the width of the grid (165 $\mathrm{m}$ for this study) and $W_{B}$ is the width of the border zone (the area trapped outside of the actual grid). The mathematical procedures for estimating $D, N, A$ and $W_{B}$ are discussed below relative to each estimation technique. Symbols have been changed from the original sources to facilitate the description and discussion of methods and the comparison of the different estimators within this paper.

\section{Standard Minimum}

The simplest estimate of density $\left(D_{S M}\right)$ is calculated using the actual number of mammals caught on the grid $\left(N_{G}\right)$ as the estimate of numbers in the area affected by the grid (Grodzinski, Pucek \& Ryszkowski, 1966). The ared of the grid $\left(A_{G}\right)$ for this procedure is 3.24 ha from Eq. 2, assuming a border zone of $1 / 2$ of the interstation distance ( $7.5 \mathrm{~m}$ in this study).

A second approach using $A_{c}$; is to estimate the numbers in the area trapped by the grid from the number caught $\left(N_{c_{i}}\right)$ using the regression method of 11 a yne (1949) or the average probability of capture procedure of Janion, Ryszkowski \& Wierzbowska (1968). Hayne's regression line technique does not work with the present data (see below). An estimate of numbers of a grid $\left(N_{G} \cdot p\right)$ is calculated from the average probability of capture $\left(P_{C}\right)$ using the methods outlined in Janion et al. (1968). Density $\left(D_{S M} \cdot p\right)$ is then calculated as $N_{G}, p_{p} / A_{G}$.

\section{Inner Square}

This technique assumes that density in the inner portion of the grid is identical to the density of the area effectively trapped by the entire grid such that

$$
D_{S Q}=\frac{N_{1}}{A_{1}}=\frac{N_{G}}{A_{S Q}}
$$

where $D_{S Q}$ is the density estimated by the Inner Square method, $N_{1}$ the number of animals captured on the inner square, $A_{1}$ the area of the inner square, $N_{G}$ the number of animals captured on the entire grid and $A_{S Q}$ the area effectively trapped by the grid (A u l a k, 1967; B u cha 1czyk \& Pucek, 1968; Adamczyk \& Ryszkowski, 1968; Hansson, 1969; Pelikan, 1970). The width of the border zone $\left(W_{B}\right)$ is calculated from $A_{S Q}$ using Eq. 2. The inner square with constant capture per area is estimated by comparing the captures on the 
outer belts of traps $\left(N_{O}\right)$ and the inner squares $\left(N_{,}\right)$to the expected captures based on the proportion of trapping stations in each region using Chi-square analyses.

Density estimates can also be calculated from a modification of the Inner Square method. In this procedure, probabilities of capture on the inner square $\left(P_{c}, I S\right)$ and grid $\left(P_{c}\right)$ are used to estimate $N_{1, P}$ and $N_{G} \cdot P$ for the respective areas. Density $\left(D_{S Q} \cdot P\right)$ is then estimated from $N_{1}, P$ and $A_{I}$ whereas area trapped by the grid $\left(A_{S Q} \cdot P\right)$ is estimated from $N_{G \cdot P}$ and $D_{S Q} \cdot P$.

\section{Assessment Line}

The Assessment Line method utilizes the change in captures per station in the area of effect in relation to captures per station outside of the area affected by removal trapping on the grid (details of the procedure in $\mathrm{Kaufman}$ et al., 1971; $\mathrm{Smith}$ et al., 1971; S $\mathrm{mith}$ et al., 1975). The width of the area of effect $\left(W_{A L}\right)$ is estimated from the intersection of two regression lines calculated for accumulated captures along the assessment lines (the first is for captures witside of the area of effect and the second for captures inside the area of effect). The total area affected by the grid $\left(A_{L A}\right)$ is then determined from Eg. 2 by substituting $W_{A L}$ for $W_{B}$. The proportion of animals removed $(R)$ from $W_{A L}$ by grid trapping is calculated from the slopes of the two regression lines such that $R=1-b_{i} / b_{0}$ where $b_{o}$ is the slope for accumulated captures over distance outside of the area of effect and $b_{i}$ the slope inside the area of effect. The population number for the area of effect $\left(N_{A L}\right)$ is equal to $N_{G} / R$ and therefore, density $\left(D_{A L}\right)$ equals $N_{A L} / A_{A L} \cdot N_{G}$ is the value for 10 days of trapping except on Boulder and Joshua II where 13 and 12 day totals, respectively, were utilized.

A second approach using assessment line data involves modification of the estimate of the proportion removed from the area of effect to adjust for differences in probability of capture inside and outside of this area. The slopes of the regression equations, which are density estimates in terms of numbers per $m$ along the assessment lines, are increased relative to probabilities of capture inside $\left(P_{c}, l_{n}\right)$ and outside $\left(P_{\mathrm{c}}\right.$. out $)$ the are of effect. An estimate of the proportion of animals removed $\left(R_{P}\right)$ is calculated from the modified values of $b_{o}$ and $b_{i}$ and used to estimate density $\left(D_{A L} \cdot P\right)$.

\section{RESULTS}

A total of 876 rodents representing 19 species v'ere caught on the 12 grids. Numbers of individuals and species per grid were quite variable 
Table 1

Numbers or rodents captured on grids (10 days) and associated assessment lines (4 davs) in California and Nevada.

\begin{tabular}{|c|c|c|c|c|c|}
\hline \multicolumn{3}{|c|}{ California } & \multicolumn{3}{|c|}{ Nevada } \\
\hline Species & Grid & Lines & Species & Grid & Lines \\
\hline \multicolumn{3}{|c|}{ Barrel } & \multicolumn{3}{|c|}{ Creosote I } \\
\hline P. formosus & 73 & 84 & P. formosus & 12 & 3 \\
\hline$D$ merriami & 14 & 5 & P. crinitus & 7 & 12 \\
\hline N. lepüda & 3 & 1 & D. merriami & 7 & 2 \\
\hline P. longimembris & 1 & 5 & O. torridus & 3 & - \\
\hline P. penicillatus & 1 & - & A. leucurus & 2 & 2 \\
\hline P. mamiculatus & 1 & - & P. manicuatus & 1 & 1 \\
\hline P. bai-eyi & - & 1 & P. longimembris & 1 & - \\
\hline A. leucurus & - & 1 & Total & 33 & 20 \\
\hline Total & 93 & 97 & & & \\
\hline
\end{tabular}

Boulder a
P. crimitus
N. lepida
P. formosus
P. fallax
A. leucurus
D. merriami
Total

$\begin{array}{rr}72 & 135 \\ 60 & 36 \\ 25 & 16 \\ 8 & 11 \\ 1 & 2 \\ 1 & \\ 167 & 200\end{array}$

\section{Chaparral I}
$P$. eremicus
N. lepida
$P$. californicus
N. fuscipes
D. agilis
P. fallax
Total

$\begin{array}{r}20 \\ 5 \\ 4 \\ 2 \\ 1 \\ \hline 32\end{array}$

\section{Chaparral II}

\section{P. eremicus}

D. agilis

P. ca'ifornicus

P. manicu'atus

R. megalotis

P. fal:ax

P. penicillatus

$P$. crinitus

N. lepida

N. fuscipes

Total
P. maniculatus
D. merriami
P. longimembris
D. agilis
$P$. penicillatus

$\begin{aligned} 135 & \text { P. formosus } \\ 36 & \text { D. merriami } \\ 16 & \text { A. leucurus } \\ 11 & \text { P. longimembris } \\ 2 & \text { O. torridus } \\ \overline{1} & \text { D. microps } \\ 200 & \text { P. crinitus } \\ & \text { P. maniculatus } \\ & \text { Total }\end{aligned}$

Creosote II

\begin{tabular}{rr}
11 & 5 \\
5 & 19 \\
4 & 3 \\
3 & 3 \\
2 & 7 \\
2 & 2 \\
1 & 6 \\
$\overline{28}$ & 1 \\
\hline
\end{tabular}

Creosote III

$\begin{array}{lrr}\text { P. longimembris } & 18 & 18 \\ \text { A. leucurus } & 9 & 3 \\ \text { O. torridus } & 4 & 4 \\ \text { D. merriami } & 2 & 10 \\ \text { D. microps } & 2 & 6 \\ \text { P. maniculatus } & 2 & -13 \\ \text { P. crinitus } & - & 1 \\ \text { N. lepida } & - & 1 \\ \text { P. formosus } & - & 56 \\ \text { Total } & 37 & \end{array}$

Joshua I
P. formosus
P. crinitus
D. merriami
A. leucurus
N. lepida
R. megalotis
P. maniculatus
Total

$\begin{array}{rr}17 & 21 \\ 10 & 18 \\ 8 & 22 \\ 2 & 1 \\ 2 & - \\ 1 & - \\ \frac{1}{40} & 64\end{array}$

Joshua II b
P. crinitus
P. formosus
P. lonaimembris
D. microps
O. torridus

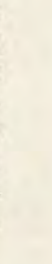


Table 1 , continued

\begin{tabular}{|c|c|c|c|c|c|}
\hline \multicolumn{3}{|l|}{ California } & \multicolumn{3}{|l|}{ Nevada } \\
\hline Species & Grid & Lines & Species & Grid & Lines \\
\hline P. fallax & 3 & 4 & D. merriami & 5 & 2 \\
\hline N. lepida & 2 & - & N. lepida & 4 & 5 \\
\hline O. torridus & 1 & 2 & A. leucurus & 4 & 2 \\
\hline P. eremicus & 1 & - & P. maniculatus & 1 & 9 \\
\hline \multirow[t]{3}{*}{ Total } & 81 & 114 & P. truei & $-\overline{100}$ & 8 \\
\hline & \multirow{2}{*}{\multicolumn{2}{|c|}{ Ocotillo }} & Total & 129 & 87 \\
\hline & & & \multicolumn{3}{|c|}{ Piñon } \\
\hline D. merriami & 9 & 14 & P. maniculatus & 43 & 51 \\
\hline P. longimembris & 3 & 3 & P. truei & 31 & 38 \\
\hline \multirow{11}{*}{ Total } & 12 & 17 & P. crinitus & 5 & 6 \\
\hline & & & A. leucurus & 3 & 7 \\
\hline & & & E. panamintinus & 3 & 5 \\
\hline & & & O. torridus & 2 & 1 \\
\hline & & & N. lepida & 1 & 4 \\
\hline & & & D. merriami & 1 & 2 \\
\hline & & & D. microps & 1 & 1 \\
\hline & & & $P$. parvus & 1 & 1 \\
\hline & & & P. formosus & - & 3 \\
\hline & & & R. mega.otis & - & 1 \\
\hline & & & Total & 91 & 120 \\
\hline
\end{tabular}

a For six species caught on Boulder, 13 day totals were $79,68,33,8,1$ and 1 , respectively.

b For ten species caught on Joshua II, 12 day totals were $61,34,19,11,9,6,4,4$, 1 and 2 , respectively.

and ranged from 12 to 167 individuals and from 2 to 10 species for 10 days of trapping (Table 1). Numbers on Joshua II and Boulder were 151 and 190 rodents for 12 and 13 days, respectively. Species composition of the small mammal communities also varied considerably among grid sites and as a result provided a wide array of conditions under which to test the density estimation techniques. Species caught on the grid and assessment lines within a site were similar such that the common species at a grid tended to be the same during both phases of trapping (Table 1). However, the less common species at a study site were often captured only on the grid or only on the assessment lines.

The 19 species captured on the grids in descending order of the total captures were 180 Perognathus formosus M e r riam, 1889 on 6 grids, 164 Peromyscus crinitus (M e r r i m, 1891) on 7 grids, 92 Peromyscus maniculatus (W a g n e r, 1845) on 7 grids, 86 Neotoma lepida Tho$\mathrm{m}$ a s, 1893 on 8 grids, 69 Dipodomys merriami Mearns, 1890 on 10 grids, 68 Peromyscus eremicus (Baird, 1858) on 3 grids, 58 Perognathus longimembris (C o u e s, 1875) on 7 grids, 33 Peromyscus truei ( $\mathrm{S}$ h uf e ld t, 1885) on 2 grids, 25 Ammospermophilus leucurus (M e r r i a m, 1889) on 7 grids, 21 Onychomys torridus (Cou e s, 1874) on 6 grids, 17 
Dipodomys agilis $\mathrm{Gambe} 1,1848$ on 3 grids, 16 Dipodomys microps (M e r r i a m, 1904) on 4 grids, 15 Perognathus fallax M er ria m, 1889 on 3 grids, 14 Peromyscus californicus (G a m be l, 1848) on 2 grids, 7 Perognathus penicillatus Woodhouse, 1852 on 3 grids, 5 Reithrodontomys megalotis (B a i $\mathrm{rd}, 1858$ ) on 2 grids, 3 Eutamias panamintinus (M e r r i m, 1893) on 1 grid, 2 Neotoma fuscipes B a i d, 1858 on 1 grid and 1 Perognathus parvus ( $\mathrm{P} \mathrm{e}$ a l e, 1848) on 1 grid. In addition, 1 Perognathus baileyi Merria m, 1894 was caught on the Barrel assessment lines.

Table 2

Accumulative per cent removal for all rodents and common species $\left(N_{G}>20\right)$ on each grid.

\begin{tabular}{|c|c|c|c|c|c|c|c|c|c|c|c|}
\hline \multirow[b]{2}{*}{ Grid } & \multirow[b]{2}{*}{ Species } & \multicolumn{10}{|c|}{ Day } \\
\hline & & 1 & 2 & 3 & 4 & 5 & 6 & 7 & 8 & 9 & 10 \\
\hline \multirow[t]{2}{*}{ Barrel } & All & 20 & 31 & 47 & 57 & 69 & 78 & 85 & 87 & 96 & 100 \\
\hline & P. formosus & 20 & 33 & 48 & 58 & 68 & 78 & 85 & 86 & 96 & 100 \\
\hline \multirow[t]{4}{*}{ Boulder } & All & 12 & 32 & 47 & 62 & 71 & 74 & 80 & 85 & 94 & 100 \\
\hline & P. crinitus & 19 & 40 & 58 & 69 & 74 & 76 & 81 & 83 & 92 & 100 \\
\hline & N. lepida & 7 & 28 & 38 & 62 & 73 & 73 & 78 & 83 & 93 & 100 \\
\hline & P. formosus & 4 & 16 & 36 & 48 & 60 & 72 & 84 & 96 & 100 & 100 \\
\hline \multirow[t]{2}{*}{ Chaparral I } & All & 47 & 50 & 59 & 62 & 81 & 84 & 88 & 88 & 94 & 100 \\
\hline & P. eremicus & 60 & 65 & 70 & 75 & 95 & 100 & 100 & 100 & 100 & 100 \\
\hline \multirow[t]{2}{*}{ Chaparral II } & All & 42 & 58 & 66 & 74 & 85 & 89 & 91 & 93 & 93 & 100 \\
\hline & P. eremicus & 49 & 64 & 70 & 79 & 83 & 85 & 87 & 92 & 92 & 100 \\
\hline \multirow[t]{2}{*}{ Juniper } & All & 30 & 36 & 48 & 54 & 58 & 59 & 73 & 82 & 94 & 100 \\
\hline & P. maniculatus & 40 & 46 & 63 & 71 & 77 & 80 & 91 & 91 & 94 & 100 \\
\hline Ocotillo & All & 25 & 50 & 58 & 58 & 75 & 83 & 92 & 92 & 92 & 100 \\
\hline Creosote I & All & 52 & 61 & 70 & 70 & 76 & 76 & 76 & 79 & 82 & 100 \\
\hline Creosote II & All & 0 & 29 & 54 & 64 & 75 & 82 & 82 & 93 & 96 & 100 \\
\hline Creosote III & All & 16 & 35 & 41 & 51 & 60 & 65 & 70 & 82 & 95 & 100 \\
\hline Joshua I & All & 38 & 45 & 52 & 70 & 75 & 90 & 90 & 95 & 98 & 100 \\
\hline \multirow[t]{3}{*}{ Joshua II } & All & 36 & 40 & 53 & 60 & 67 & 74 & 78 & 86 & 88 & 100 \\
\hline & P. crinitus & 42 & 44 & 54 & 58 & 64 & 70 & 72 & 78 & 82 & 100 \\
\hline & P. formosus & 42 & 45 & 52 & 55 & 64 & 71 & 77 & 90 & 90 & 100 \\
\hline \multirow{3}{*}{ Piñon } & All & 11 & 20 & 28 & 34 & 44 & 52 & 58 & 70 & 86 & 100 \\
\hline & P. maniculatus & 12 & 19 & 26 & 33 & 42 & 46 & 51 & 60 & 79 & 100 \\
\hline & P. truei & 13 & 16 & 26 & 32 & 36 & 52 & 61 & 77 & 90 & 100 \\
\hline All & All & 26 & 38 & 50 & 59 & 68 & 73 & 79 & 85 & 92 & 100 \\
\hline
\end{tabular}

\section{Rate of Removal}

Removal rates for all captures were compared using the accumulative per cent removal with the 10 day total as 100 per cent. The per cent removal for the first day varied from 0 to $52 \%$ with $25 \%$ or more removed the first day on seven of the 12 grids (Table 2). The average removal during day 1 for all grids combined was over $25 \%$. Regardless of the first day's capture rate, the per cent captures by day 5 were 
greater than $50 \%$ on 11 of the 12 grids. The Piñon grid was the only trap site for which the number of captures during the last five days exceeded that of the first five days.

Even though the patterns of rate of removal for all species combined were similar across grids, inter- and intrageneric differences in removal rates were apparent (Table 2). Per cent removal of common species on individual grids indicated a wide variability in trappability on the first day of trapping $(4-60 \%)$ that persisted through the fifth day $(36-95 \%)$. This wide range of variability was recorded for Peromyscus ( $P$. truei was $36 \%$, P. maniculatus $42-77 \%$, P. crinitus $64-74 \%$ and $P$. eremicus

Table 3

Accumulative per cent removal for five genera and 13 species using data pooled from captures on all 12 grids.

\begin{tabular}{|c|c|c|c|c|c|c|c|c|c|c|c|c|}
\hline \multirow[b]{2}{*}{ Genus } & \multirow[b]{2}{*}{ Species } & \multirow[b]{2}{*}{$\mathrm{N}$} & \multicolumn{9}{|c|}{ Day } & \multirow[b]{2}{*}{10} \\
\hline & & & 1 & 2 & 3 & 4 & 5 & 6 & 7 & 8 & 9 & \\
\hline Peromyscus & $\begin{array}{l}\text { All } \\
\text { crinitus } \\
\text { maniculatus } \\
\text { eremicus } \\
\text { truei } \\
\text { californicus }\end{array}$ & $\begin{array}{r}351 \\
146 \\
92 \\
68 \\
31 \\
14\end{array}$ & $\begin{array}{l}31 \\
27 \\
26 \\
51 \\
13 \\
43\end{array}$ & $\begin{array}{l}42 \\
42 \\
32 \\
65 \\
16 \\
50\end{array}$ & $\begin{array}{l}53 \\
55 \\
42 \\
71 \\
26 \\
71\end{array}$ & $\begin{array}{l}60 \\
62 \\
51 \\
78 \\
32 \\
71\end{array}$ & $\begin{array}{l}68 \\
68 \\
61 \\
87 \\
36 \\
86\end{array}$ & $\begin{array}{l}72 \\
71 \\
65 \\
90 \\
52 \\
93\end{array}$ & $\begin{array}{l}77 \\
75 \\
72 \\
91 \\
61 \\
93\end{array}$ & $\begin{array}{l}81 \\
79 \\
77 \\
94 \\
77 \\
93\end{array}$ & $\begin{array}{l}89 \\
86 \\
88 \\
94 \\
90 \\
93\end{array}$ & $\begin{array}{l}100 \\
100 \\
100 \\
100 \\
100 \\
100\end{array}$ \\
\hline Perognathus & $\begin{array}{l}\text { All } \\
\text { formosus } \\
\text { longimembris } \\
\text { fa'lax }\end{array}$ & $\begin{array}{r}250 \\
169 \\
58 \\
15\end{array}$ & $\begin{array}{l}23 \\
24 \\
22 \\
20\end{array}$ & $\begin{array}{l}34 \\
33 \\
38 \\
33\end{array}$ & $\begin{array}{l}48 \\
48 \\
50 \\
47\end{array}$ & $\begin{array}{l}58 \\
57 \\
64 \\
60\end{array}$ & $\begin{array}{l}67 \\
67 \\
69 \\
67\end{array}$ & $\begin{array}{l}74 \\
76 \\
72 \\
67\end{array}$ & $\begin{array}{l}80 \\
82 \\
74 \\
87\end{array}$ & $\begin{array}{l}88 \\
89 \\
88 \\
87\end{array}$ & $\begin{array}{r}96 \\
95 \\
97 \\
100\end{array}$ & $\begin{array}{l}100 \\
100 \\
100 \\
100\end{array}$ \\
\hline Dipodomys & $\begin{array}{l}\text { All } \\
\text { merriami } \\
\text { agilis } \\
\text { microps }\end{array}$ & $\begin{array}{l}98 \\
68 \\
17 \\
13\end{array}$ & $\begin{array}{r}29 \\
35 \\
18 \\
8\end{array}$ & $\begin{array}{r}41 \\
47 \\
41 \\
8\end{array}$ & $\begin{array}{l}54 \\
59 \\
47 \\
38\end{array}$ & $\begin{array}{l}61 \\
66 \\
47 \\
54\end{array}$ & $\begin{array}{l}68 \\
71 \\
65 \\
62\end{array}$ & $\begin{array}{l}77 \\
76 \\
65 \\
92\end{array}$ & $\begin{array}{r}84 \\
82 \\
76 \\
100\end{array}$ & $\begin{array}{r}90 \\
91 \\
76 \\
100\end{array}$ & $\begin{array}{r}95 \\
96 \\
88 \\
100\end{array}$ & $\begin{array}{l}100 \\
100 \\
100 \\
100\end{array}$ \\
\hline Neotoma & lepida & 78 & 13 & 29 & 38 & 56 & 68 & 69 & 77 & 83 & 91 & 100 \\
\hline $\begin{array}{l}\text { Ammospermo- } \\
\text { philus }\end{array}$ & leucurus & 25 & 0 & 32 & 44 & 56 & 64 & 76 & 80 & 80 & 92 & 100 \\
\hline All & All & 831 & 26 & 38 & 50 & 59 & 68 & 73 & 79 & 85 & 92 & 100 \\
\hline
\end{tabular}

$83-95 \%)$. Removal rates of $P$. formosus $(60-68 \%)$ and $N$. lepida $(73 \%)$ were similar to the overall average for all captures.

Another comparison of removal rates between species and genera which avoids individual conditions of each grid was made by pooling across all grids (Table 3). Examination of pooled removal rates for the five common genera indicated that rates were similar during the days 4-10 of trapping although differences were considerable during the first three days. The degree of intrageneric variability in removal rate was quite different within the three genera examined (Table 3 ). The greatest intrageneric variability in rate of removal occurred among the 
three species of Dipodomys with the least variability among the three species of Perognathus.

\section{Density Estimation}

$\mathrm{St}$ andard Minimum. Density estimates based on the Standard Minimum area of the grid $\left(D_{S M}\right)$ for all species combined and six common species were calculated for days 2 through 10 for each grid (Tables 4 \& 5). Comparison of these values for any single day demonstrates the considerable variability in density of rodents among the study areas. The capture of animals throughout the trapping period resulted in an increase in the estimate of $D_{S M}$ with time. Therefore, unless the appropriate length of the trapping period is known these estimates are good only as relative estimates and are presented here for comparison to other estimates discussed below.

Estimation of numbers in the area trapped by the grid, rather than use of the number of captures, has been tried using two basic methods, the Hayne regression technique (1949) when probalility of capture is relatively constant during the entire trapping period and the average probability of capture technique of Janion et al. (1968) when probability of capture fluctuates during the trapping period. The traps were not prebaited and consequently daily probability of capture fluctuated so that Hayne's procedure would not work with the present data. Calculation of average probability of capture $\left(P_{c}\right.$ from $\mathrm{Janion}$ et al., 1968) should be useful for calculation of numbers under these conditions. Values of $P_{c}$ were calculated for all species combined for days 2 through 10 for each of the grids as well as for all grids (Table 4). Probabilities of capture were quite variable both among grids as well as among days for any single grid. For example, $P_{c}$ for day 10 ranged from 0.08 to 0.29 with the 10 day value for Piñon not calculable. Daily variability in $P_{c}$ is also demonstrated in Table 4 with at least one daily value not calculable for five of the 12 grids. On the remaining seven grids, $P_{c}$ values were greatest on day 2 with a decrease over time resulting in the smallest $P_{c}$ occurring on day 10 for 5 of the 7 grids. For all grids combined, the daily values of $P_{c}$ was the greatest on day 2 and decreased to the minimum value on day 10. Densities $\left(D_{S M \cdot P}\right)$ for day 10 (11 grids) were all higher than $D_{S M}$ (Mean $D_{S M \cdot P}=126 \%$ Mean $D_{S M}$, range 103 $177 \%$ ) since not all animals on the grid area were caught by day 10 .

Average probabilities of capture and corresponding density estimates were calculated for six rodent species that were common $\left(N_{G} \geqslant 30\right)$ on one or more grids (Table 5). Values of $P_{c}$ of the individual species for day 10 had a similar range $(0.10-0.31)$ as for all species combined 
Table 4

Densities in animals/ha $\left(D_{S M}, D_{S M} . P\right)$ and probabilities of capture $\left(P_{c}\right)$ for days 2 through 10 using the Standard Minimum technique.

\begin{tabular}{|c|c|c|c|c|c|c|c|c|c|c|}
\hline Grid & Variable & 2 & 3 & 4 & 5 & Day ${ }_{6}$ & 7 & 8 & 9 & 1 \\
\hline Barrel & $\begin{array}{l}D_{S M} \\
D_{S M} \cdot P \\
P_{c}\end{array}$ & $\begin{array}{r}9.0 \\
12.4 \\
0.47\end{array}$ & $\begin{array}{l}13.6 \\
39.8 \\
0.13\end{array}$ & $\begin{array}{l}16.4 \\
31.1 \\
0.17\end{array}$ & \begin{tabular}{l|l}
19.8 \\
41.8 \\
0.12
\end{tabular} & $\begin{array}{l}22.5 \\
42.1 \\
0.12\end{array}$ & $\begin{array}{l}24.4 \\
37.4 \\
0.14\end{array}$ & $\begin{array}{l}25.0 \\
32.3 \\
0.17\end{array}$ & $\begin{array}{l}27.5 \\
37.0 \\
0.14\end{array}$ & $\begin{array}{l}28.7 \\
36.9 \\
0.14\end{array}$ \\
\hline Boulder & $\begin{array}{l}D_{S M} \\
D_{S M} \cdot P \\
P_{c}\end{array}$ & $\begin{array}{r}16.4 \\
-\end{array}$ & $\begin{array}{r}24.1 \\
-\end{array}$ & $\begin{array}{r}32.1 \\
-\end{array}$ & \begin{tabular}{r|r}
36.4 & \\
106.8 & \\
0.08 &
\end{tabular} & $\begin{array}{l}38.0 \\
56.4 \\
0.17\end{array}$ & $\begin{array}{l}41.4 \\
58.7 \\
0.16\end{array}$ & $\begin{array}{l}43.8 \\
56.6 \\
0.17\end{array}$ & $\begin{array}{l}48.5 \\
67.8 \\
0.13\end{array}$ & $\begin{array}{l}51.5 \\
71.5 \\
0.12\end{array}$ \\
\hline Chaparral I & $\begin{array}{l}D_{S M} \\
D_{S M} \cdot P \\
P_{c}\end{array}$ & $\begin{array}{r}4.9 \\
5.0 \\
0.93\end{array}$ & $\begin{array}{r}5.9 \\
6.0 \\
0.68\end{array}$ & $\begin{array}{r}6.2 \\
6.3 \\
0.64\end{array}$ & $\begin{array}{r}8.0 \\
9.5 \\
0.31\end{array}$ & $\begin{array}{r}8.3 \\
9.2 \\
0.33\end{array}$ & $\begin{array}{r}8.6 \\
9.3 \\
0.32\end{array}$ & $\begin{array}{r}8.6 \\
8.9 \\
0.35\end{array}$ & $\begin{array}{r}9.3 \\
9.4 \\
0.29\end{array}$ & $\begin{array}{r}9.9 \\
10.6 \\
0.24\end{array}$ \\
\hline Chaparral II & $\begin{array}{l}D_{S M} \\
D_{S M} \cdot P \\
P_{c}\end{array}$ & $\begin{array}{l}15.7 \\
18.4 \\
0.62\end{array}$ & $\begin{array}{l}17.9 \\
22.3 \\
0.58\end{array}$ & $\begin{array}{l}20.1 \\
21.5 \\
0.49\end{array}$ & $\begin{array}{l}23.1 \\
25.9 \\
0.36\end{array}$ & $\begin{array}{l}24.1 \\
25.9 \\
0.36\end{array}$ & $\begin{array}{l}24.7 \\
25.8 \\
0.36\end{array}$ & & $\begin{array}{l}25.3 \\
25.7 \\
0.37\end{array}$ & $\begin{array}{l}27.2 \\
28.1 \\
0.29\end{array}$ \\
\hline Juniper & $\begin{array}{l}D_{S M} \\
D_{S M} \cdot P \\
P_{c}\end{array}$ & $\begin{array}{r}9.0 \\
9.4 \\
0.79\end{array}$ & $\begin{array}{l}12.0 \\
14.8 \\
0.43\end{array}$ & & & & & & & 25.0 \\
\hline Ocotillo & $\begin{array}{l}D_{S M} \\
D_{S M} \cdot P \\
P_{c}\end{array}$ & $\frac{1.8}{-}$ & $\begin{array}{r}2.2 \\
2.9 \\
0.36\end{array}$ & $\begin{array}{r}2.2 \\
2.3 \\
0.51\end{array}$ & $\begin{array}{r}2.8 \\
3.6 \\
0.25\end{array}$ & $\begin{array}{r}3.1 \\
4.0 \\
0.22\end{array}$ & $\begin{array}{r}3.4 \\
4.3 \\
0.20\end{array}$ & $\begin{array}{r}3.4 \\
3.8 \\
0.24\end{array}$ & $\begin{array}{r}3.4 \\
3.6 \\
0.26\end{array}$ & $\begin{array}{r}3.7 \\
4.1 \\
0.21\end{array}$ \\
\hline Creosote I & $\begin{array}{l}D_{S M} \\
D_{S M} \cdot P \\
P_{c}\end{array}$ & $\begin{array}{r}6.2 \\
6.4 \\
0.82\end{array}$ & $\begin{array}{r}7.1 \\
7.4 \\
0.64\end{array}$ & $\begin{array}{r}7.1 \\
7.2 \\
0.70\end{array}$ & $\begin{array}{r}7.7 \\
7.8 \\
0.57\end{array}$ & $\begin{array}{r}7.7 \\
7.7 \\
0.58\end{array}$ & $\begin{array}{r}7.7 \\
7.7 \\
0.59\end{array}$ & $\begin{array}{r}8.0 \\
8.1 \\
0.52\end{array}$ & $\begin{array}{r}8.3 \\
8.4 \\
0.45\end{array}$ & $\begin{array}{l}10.2 \\
11.1 \\
0.22\end{array}$ \\
\hline Creosote II & $\begin{array}{l}D_{S M} \\
D_{S M}, P \\
P_{c}\end{array}$ & $\frac{2.5}{-}$ & $\frac{4.6}{-}$ & $\frac{5.6}{-}$ & $\frac{6.5}{-}$ & $\begin{array}{r}7.1 \\
22.9 \\
0.06\end{array}$ & $\begin{array}{r}7.1 \\
10.1 \\
0.16\end{array}$ & $\begin{array}{r}8.0 \\
12.5 \\
0.12\end{array}$ & $\begin{array}{r}8.3 \\
13.6 \\
0.10\end{array}$ & $\begin{array}{r}8.6 \\
10.8 \\
0.15\end{array}$ \\
\hline Creosote III & $\begin{array}{l}D_{S M} \\
D_{S M} \cdot P \\
P_{c}\end{array}$ & $\frac{4.0}{-}$ & $\begin{array}{r}4.6 \\
6.5 \\
0.34\end{array}$ & $\begin{array}{r}5.9 \\
9.6 \\
0.21\end{array}$ & $\begin{array}{r}6.8 \\
10.4 \\
0.19\end{array}$ & $\begin{array}{r}7.4 \\
10.0 \\
0.20\end{array}$ & $\begin{array}{r}8.0 \\
10.4 \\
0.19\end{array}$ & $\begin{array}{r}9.3 \\
14.4 \\
0.12\end{array}$ & $\begin{array}{l}10.8 \\
25.3 \\
0.06\end{array}$ & $\begin{array}{l}11.4 \\
20.2 \\
0.08\end{array}$ \\
\hline Joshua I & $\begin{array}{l}D_{S M} \\
D_{S M} \cdot P \\
P_{c}\end{array}$ & $\begin{array}{r}5.6 \\
5.8 \\
0.80\end{array}$ & $\begin{array}{r}6.5 \\
6.8 \\
0.63\end{array}$ & $\begin{array}{r}8.6 \\
11.4 \\
0.30\end{array}$ & $\begin{array}{r}9.3 \\
10.8 \\
0.32\end{array}$ & $\begin{array}{l}11.1 \\
15.5 \\
0.19\end{array}$ & $\begin{array}{l}11.1 \\
12.7 \\
0.26\end{array}$ & $\begin{array}{l}11.7 \\
13.2 \\
0.24\end{array}$ & $\begin{array}{l}12.0 \\
13.1 \\
0.24\end{array}$ & $\begin{array}{l}12.3 \\
13.2 \\
0.24\end{array}$ \\
\hline Joshua II & $\begin{array}{l}D_{S M} \\
D_{S M} \cdot P \\
P_{c}\end{array}$ & $\begin{array}{l}15.7 \\
15.8 \\
0.92\end{array}$ & $\begin{array}{l}21.0 \\
23.8 \\
0.51\end{array}$ & $\begin{array}{l}23.8 \\
26.4 \\
0.44\end{array}$ & $\begin{array}{l}26.5 \\
29.7 \\
0.36\end{array}$ & $\begin{array}{l}29.3 \\
33.2 \\
0.30\end{array}$ & $\begin{array}{l}31.2 \\
34.7 \\
0.28\end{array}$ & $\begin{array}{l}34.3 \\
36.7 \\
0.22\end{array}$ & $\begin{array}{l}35.2 \\
39.4 \\
0.22\end{array}$ & $\begin{array}{l}39.8 \\
49.6 \\
0.15\end{array}$ \\
\hline Piñon & $\begin{array}{l}D_{S M} \\
D_{S M} \cdot P \\
P_{c}\end{array}$ & $\begin{array}{r}5.6 \\
15.4 \\
0.20\end{array}$ & $\begin{array}{r}7.7 \\
18.0 \\
0.17\end{array}$ & $\begin{array}{r}9.6 \\
19.0 \\
0.16\end{array}$ & $\begin{array}{l}12.3 \\
54.6 \\
0.05\end{array}$ & $\begin{array}{l}14.5 \\
66.8 \\
0.04\end{array}$ & $\begin{array}{l}16.4 \\
46.5 \\
0.06\end{array}$ & $\begin{array}{r}19.8 \\
-\end{array}$ & $\begin{array}{r}24.1 \\
-\end{array}$ & ${ }^{28.1}$ \\
\hline All & $\begin{array}{l}D_{S M} \\
D_{S M} \cdot P \\
P_{c}\end{array}$ & $\begin{array}{r}8.0 \\
10.2 \\
0.54\end{array}$ & $\begin{array}{l}10.6 \\
14.9 \\
0.34\end{array}$ & $\begin{array}{l}12.6 \\
16.9 \\
0.29\end{array}$ & $\begin{array}{l}14.5 \\
19.4 \\
0.24\end{array}$ & $\begin{array}{l}15.7 \\
19.4 \\
0.24\end{array}$ & $\begin{array}{l}16.8 \\
20.4 \\
0.22\end{array}$ & $\begin{array}{l}18.1 \\
21.8 \\
0.20\end{array}$ & $\begin{array}{l}19.7 \\
24.2 \\
0.17\end{array}$ & $\begin{array}{l}21.4 \\
27.4 \\
0.14\end{array}$ \\
\hline
\end{tabular}

Note. - Dash indicates that probability of capture could not be calculated.

(Tables 4 \& 5). Estimates of density would vary considerably depending on whether $P_{c}$ for individual species or $P_{c}$ for all species combined are used to compute density. Densities for common species should be calculated from the species $P_{c}$ value. This qualification places limitations 
on the use of this procedure since densities of many species are too low for estimation by $P_{c}$.

Consideration of the temporal changes in $D_{S M} \cdot p$ in Tables 4,5 indicates that $D_{S M} \cdot{ }_{P}$ like $D_{S M}$ does not plateau at some rather constant density.

Table 5

Densities in animals/ha $\left(D_{S M}, D_{S M} \cdot P\right)$ and probabilities of capture $\left(P_{c}\right)$ of six common species $\left(N_{G}>30\right)$ for days 2 through 10 using the Standard Minimum technique.

\begin{tabular}{|c|c|c|c|c|c|c|c|c|c|c|}
\hline \multirow{2}{*}{$\begin{array}{c}\text { Species } \\
\text { Grid }\end{array}$} & \multirow[b]{2}{*}{ Variable } & \multicolumn{9}{|c|}{ Day } \\
\hline & & 2 & 3 & 4 & 5 & 6 & 7 & 8 & 9 & 10 \\
\hline \multicolumn{11}{|l|}{ P. formosus } \\
\hline Barrel & $\begin{array}{l}D_{S M} \\
D_{S M} \cdot P \\
P_{c}\end{array}$ & $\begin{array}{r}7.4 \\
11.6 \\
0.40\end{array}$ & $\begin{array}{l}10.8 \\
26.5 \\
0.16\end{array}$ & $\begin{array}{l}13.0 \\
22.7 \\
0.19\end{array}$ & $\begin{array}{l}15.4 \\
27.7 \\
0.15\end{array}$ & $\begin{array}{l}17.6 \\
31.0 \\
0.13\end{array}$ & $\begin{array}{l}19.1 \\
29.4 \\
0.14\end{array}$ & $\begin{array}{l}19.4 \\
24.4 \\
0.18\end{array}$ & $\begin{array}{l}21.6 \\
29.1 \\
0.14\end{array}$ & $\begin{array}{l}22.5 \\
28.9 \\
0.14\end{array}$ \\
\hline \multirow[t]{3}{*}{ Joshua II } & $D_{S M}$ & 4.3 & 4.9 & 5.2 & 6.2 & 6.8 & 7.4 & 8.6 & 8.6 & 9.6 \\
\hline & $D_{S M} \cdot P$ & 4.4 & 5.0 & 5.3 & 6.6 & 7.3 & 8.1 & 10.9 & 9.7 & 11.6 \\
\hline & $P_{c}$ & 0.92 & 0.73 & 0.65 & 0.43 & 0.35 & 0.29 & 0.18 & 0.22 & 0.16 \\
\hline \\
\hline \multirow{3}{*}{ Boulder } & $D_{S M}$ & 9.0 & 13.0 & 15.4 & 16.4 & 17.0 & 17.9 & 18.5 & 20.4 & 22.2 \\
\hline & $D_{S M} \cdot P$ & - & 220.4 & 32.3 & 21.5 & 19.7 & 19.9 & 20.2 & 23.1 & 26.3 \\
\hline & $P_{c}$ & - & 0.02 & 0.15 & 0.25 & 0.28 & 0.28 & 0.27 & 0.21 & 0.17 \\
\hline \multirow[t]{3}{*}{ Joshua II } & $D_{S M}$ & 6.8 & 8.3 & 9.0 & 9.9 & 10.8 & 11.1 & 12.0 & 12.7 & 15.4 \\
\hline & $D_{S M} \cdot P$ & 6.8 & 8.7 & 9.2 & 10.3 & 11.5 & 11.5 & 12.8 & 13.5 & 20.5 \\
\hline & $P_{c}$ & 0.95 & 0.65 & 0.59 & 0.47 & 0.38 & 0.38 & 0.30 & 0.26 & 0.13 \\
\hline \multicolumn{11}{|l|}{ P. maniculatus } \\
\hline \multirow[t]{3}{*}{ Juniper } & ESM & 4.9 & 6.8 & 7.7 & 8.3 & 8.6 & 9.9 & 9.9 & 10.2 & 10.8 \\
\hline & $D_{S M} \cdot P$ & 5.0 & 8.2 & 8.9 & 9.3 & 9.2 & 11.2 & 10.6 & 10.7 & 11.5 \\
\hline & $P_{c}$ & 0.86 & 0.44 & 0.40 & 0.37 & 0.38 & 0.26 & 0.29 & 0.28 & 0.24 \\
\hline \multirow[t]{3}{*}{ Piñon } & $D_{S M}$ & 2.5 & 3.4 & 4.3 & 5.6 & 6.2 & 6.8 & 8.0 & 10.5 & 13.3 \\
\hline & $D_{S M} \cdot P$ & 3.9 & 6.0 & 8.6 & 14.6 & 13.2 & 12.2 & 23.8 & - & - \\
\hline & $P_{c}$ & 0.40 & 0.24 & 0.16 & 0.05 & 0.10 & 0.11 & 0.05 & - & - \\
\hline \multicolumn{11}{|l|}{ P. eremicus } \\
\hline \multirow[t]{3}{*}{ Chaparral II } & $D_{S M}$ & 9.3 & 10.2 & 11.4 & 12.0 & 12.3 & 12.7 & 13.3 & 13.3 & 14,5 \\
\hline & DSM. & 10.2 & 10.6 & 12.0 & 12.4 & 12.6 & 12.8 & 13.5 & 13.4 & 14.9 \\
\hline & $P_{c}$ & 0.70 & 0.66 & 0.53 & 0.50 & 0.48 & 0.46 & 0.40 & 0.42 & 0.31 \\
\hline \multirow{3}{*}{$\begin{array}{l}\text { P. truei } \\
\text { Piñon }\end{array}$} & & & & & & & & & & \\
\hline & DSM & 1.5 & 2.5 & 3.1 & 3.4 & 4.9 & 5.9 & 7.4 & 8.6 & 9.6 \\
\hline & $\begin{array}{l}D_{S M} \cdot P \\
P\end{array}$ & $\begin{array}{r}1.6 \\
0.75\end{array}$ & $\begin{array}{r}5.8 \\
0.17\end{array}$ & $\begin{array}{r}6.5 \\
0.15\end{array}$ & 3.4 & - & - & - & - & - \\
\hline \multirow{4}{*}{$\begin{array}{l}N . \text { lenida } \\
\text { Boulder }\end{array}$} & & & 0.17 & 0.15 & 0.21 & & & & & 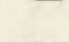 \\
\hline & $D_{S M}$ & 5.2 & 7.1 & 11.4 & 13.6 & 13.6 & 14.5 & 15.4 & 17.3 & 18.5 \\
\hline & $D_{S M} \cdot P$ & - & - & - & - & 27.0 & 22.3 & 21.2 & 26.6 & 28.4 \\
\hline & $P_{\text {n }}$ & - & - & - & - & 0.11 & 0.14 & 0.15 & 0.11 & 0.10 \\
\hline
\end{tabular}

Note. - Dash indicates that probability of capture could not be calculated.

This suggests that $D_{S M} \cdot P$ is also a relative density index although the relative numbers may be more highly correlated to the true densities than are $D_{S M}$ since differences in probability of capture are theoretically adjusted for in $D_{S M} \cdot P$. Therefore, to use $D_{S M} \cdot P$ as an estimator of absolute rather than relative density, considerable understanding of the 
Table 6

Densities in animals/ha $(D S Q, D S Q, P)$ and areas effectively trapped $\left(A_{S Q}, A_{S Q} \cdot P\right.$ ) for days 2 through 10 using the Inner Square technique.

\begin{tabular}{|c|c|c|c|c|c|c|c|c|c|c|}
\hline \multirow[b]{2}{*}{ Grid } & \multirow[b]{2}{*}{ Variable } & \multicolumn{9}{|c|}{ Day } \\
\hline & & 2 & 3 & 4 & 5 & 6 & 7 & 8 & 9 & 10 \\
\hline Barrel & $\begin{array}{l}D_{S Q} \\
A_{S Q} \\
D_{S Q} \cdot P \\
A_{S Q} \cdot P\end{array}$ & $\begin{array}{r}10.2 \\
2.8 \\
12.7 \\
3.2\end{array}$ & $\begin{array}{r}14.7 \\
3.0 \\
26.1 \\
4.9\end{array}$ & $\begin{array}{r}16.7 \\
3.1 \\
23.1 \\
4.4\end{array}$ & $\begin{array}{r}19.6 \\
3.3 \\
26.8 \\
5.1\end{array}$ & $\begin{array}{r}21.8 \\
3.4 \\
29.5 \\
4.6\end{array}$ & $\begin{array}{r}24.0 \\
3.3 \\
32.0 \\
3.8\end{array}$ & \begin{tabular}{r|r}
24.4 \\
3.3 \\
28.8 \\
3.6
\end{tabular} & $\begin{array}{r}26.2 \\
3.4 \\
31.5 \\
3.8\end{array}$ & $\begin{array}{r}27.6 \\
3.4 \\
32.6 \\
3.7\end{array}$ \\
\hline Boulder & $\begin{array}{l}D_{S Q} \\
A_{S Q} \\
D_{S Q} \cdot P \\
A_{S Q} \cdot P\end{array}$ & $\begin{array}{r}13.8 \\
3.8 \\
- \\
-\end{array}$ & $\begin{array}{c}20.0^{\mathrm{b}} \\
4.0 \\
-\end{array}$ & $\begin{array}{c}24.9 \mathrm{~b} \\
4.2 \\
320.6 \\
-\end{array}$ & $\begin{array}{c}28.9^{\mathrm{b}} \\
4.1 \\
76.8 \\
4.5\end{array}$ & $\begin{array}{c}30.7 \mathrm{~b} \\
4.0 \\
47.3 \\
3.9\end{array}$ & $\begin{array}{c}32.9^{\mathrm{b}} \\
4.1 \\
45.2 \\
4.2\end{array}$ & $\begin{array}{c}33.8^{\mathrm{b}} \\
4.2 \\
40.6 \\
4.5\end{array}$ & $\begin{array}{c}35.6^{\mathrm{b}} \\
4.4 \\
41.8 \\
5.3\end{array}$ & $\begin{array}{c}36.9 \mathrm{~b} \\
4.5 \\
42.0 \\
5.5\end{array}$ \\
\hline Chaparral I & $\begin{array}{l}D_{S Q} \\
A_{S Q} \\
D_{S Q} \cdot P \\
A_{S Q} \cdot P\end{array}$ & $\begin{array}{l}4.9 \\
3.3 \\
4.9 \\
3.3 \\
\end{array}$ & $\begin{array}{l}5.3 \\
3.6 \\
5.4 \\
3.6\end{array}$ & $\begin{array}{l}5.8 \\
3.5 \\
5.9 \\
3.4\end{array}$ & $\begin{array}{l}7.1 \\
3.7 \\
7.8 \\
3.9\end{array}$ & $\begin{array}{l}7.6 \\
3.6 \\
8.1 \\
3.7\end{array}$ & $\begin{array}{l}7.6 \\
3.7 \\
7.8 \\
3.8 \\
\end{array}$ & $\begin{array}{l}7.6 \\
3.7 \\
7.7 \\
3.8\end{array}$ & $\begin{array}{l}7.6 \\
4.0 \\
7.6 \\
4.0\end{array}$ & $\begin{array}{l}8.4 \\
3.8 \\
8.8 \\
3.9\end{array}$ \\
\hline Chaparral II & $\begin{array}{l}D_{S Q} \\
A_{S Q} \\
D_{S Q} \cdot P \\
A_{Q S} \cdot P\end{array}$ & $\begin{array}{r}14.7 \\
3.5 \\
15.8 \\
3.8\end{array}$ & $\begin{array}{r}16.0 \\
3.6 \\
16.5 \\
4.4\end{array}$ & $\begin{array}{r}16.9 \\
3.8 \\
17.2 \\
4.1\end{array}$ & $\begin{array}{c}18.7^{\mathrm{a}} \\
4.0 \\
19.3 \\
4.4\end{array}$ & $\begin{array}{c}19.1^{\mathrm{b}} \\
4.1^{1} \\
19.5 \\
4.3\end{array}$ & $\begin{array}{c}19.6^{\mathrm{b}} \\
4.1 \\
19.8 \\
4.2\end{array}$ & $\begin{array}{c}20.0^{\mathrm{b}} \\
4.1 \\
20.2 \\
4.2\end{array}$ & $\begin{array}{c}20.0^{\mathrm{b}} \\
4.1 \\
20.1 \\
4.1\end{array}$ & $\begin{array}{c}20.4^{\mathrm{b}} \\
4.3 \\
20.5 \\
4.4\end{array}$ \\
\hline Juniper & $\begin{array}{l}D_{S Q} \\
A_{S Q} \\
D_{S Q} \cdot P \\
A_{S Q} \cdot P\end{array}$ & $\begin{array}{l}7.1 \\
4.1 \\
7.5 \\
4.0\end{array}$ & $\begin{array}{c}9.3 \mathrm{a} \\
4.2 \\
11.2 \\
4.3\end{array}$ & $\begin{array}{r}11.1 \\
4.0 \\
13.7 \\
3.7\end{array}$ & $\begin{array}{r}12.0 \\
3.9 \\
13.7 \\
3.7\end{array}$ & $\begin{array}{c}12.0^{\mathrm{a}} \\
4.0 \\
12.6 \\
4.0\end{array}$ & $\begin{array}{c}14.7^{\mathrm{a}} \\
4.0 \\
18.1 \\
4.0\end{array}$ & $\begin{array}{c}16.9^{\mathrm{a}} \\
3.9 \\
24.1 \\
3.6\end{array}$ & $\begin{array}{c}18.7^{\mathrm{b}} \\
4.1 \\
28.1 \\
4.3\end{array}$ & $\begin{array}{c}19.6 \text { b } \\
4.1 \\
27.1 \\
4.6\end{array}$ \\
\hline Ocotillo & $\begin{array}{l}D_{S Q} \\
A_{S Q} \\
D_{Q S} \cdot P \\
A_{Q S \cdot P} \cdot P\end{array}$ & $\begin{array}{l}1.8 \\
3.4 \\
- \\
-\end{array}$ & $\begin{array}{l}2.2 \\
3.2 \\
- \\
-\end{array}$ & $\begin{array}{l}2.2 \\
3.2 \\
2.8 \\
2.6\end{array}$ & $\begin{array}{r}3.1 \\
2.9 \\
10.2 \\
1.2\end{array}$ & $\begin{array}{l}3.1 \\
3.2 \\
4.2 \\
3.1\end{array}$ & $\begin{array}{l}3.6 \\
3.1 \\
5.2 \\
2.7\end{array}$ & $\begin{array}{l}3.6 \\
3.1 \\
4.3 \\
2.9\end{array}$ & $\begin{array}{l}3.6 \\
3.1 \\
3.9 \\
3.0\end{array}$ & $\begin{array}{l}4.0 \\
3.0 \\
4.8 \\
2.8\end{array}$ \\
\hline Creosote I & $\begin{array}{l}D_{S Q} \\
A_{S Q} \\
D_{S Q} \cdot P \\
A_{S Q} \cdot P\end{array}$ & $\begin{array}{l}5.8 \\
3.5 \\
6.0 \\
3.4\end{array}$ & $\begin{array}{l}6.7 \\
3.4 \\
7.0 \\
3.4\end{array}$ & $\begin{array}{l}6.7 \\
3.4 \\
6.7 \\
3.5\end{array}$ & $\begin{array}{l}7.1 \\
3.5 \\
7.2 \\
3.5\end{array}$ & $\begin{array}{l}7.1 \\
3.5 \\
7.2 \\
3.5 \\
\end{array}$ & $\begin{array}{l}7.1 \\
3.5 \\
7.1 \\
3.5\end{array}$ & $\begin{array}{l}7.1 \\
3.7 \\
7.1 \\
3.7\end{array}$ & $\begin{array}{l}7.1 \\
3.8 \\
7.1 \\
3.8\end{array}$ & $\begin{array}{l}7.6= \\
4.4 \\
7.6 \\
4.7\end{array}$ \\
\hline Creosote II & $\begin{array}{l}D_{S Q} \\
A_{S Q} \\
D_{S Q} \cdot P \\
A_{S Q} \cdot P\end{array}$ & $\begin{array}{l}1.3 \\
6.0 \\
- \\
-\end{array}$ & $\begin{array}{l}3.1 \\
4.8 \\
- \\
-\end{array}$ & $\begin{array}{l}4.0 \\
4.5 \\
- \\
-\end{array}$ & $\begin{array}{l}5.3 \\
3.9 \\
- \\
-\end{array}$ & $\begin{array}{l}5.8 \\
4.0 \\
- \\
-\end{array}$ & \begin{tabular}{r|}
5.8 \\
4.0 \\
12.0 \\
2.7
\end{tabular} & $\begin{array}{r}6.2 \\
4.2 \\
10.9 \\
3.7\end{array}$ & $\begin{array}{l}6.2^{a} \\
4.3 \\
8.1 \\
5.4\end{array}$ & $\begin{array}{l}6.7 \\
4.2 \\
8.6 \\
4.6\end{array}$ \\
\hline Creosote III & $\begin{array}{l}D_{S Q} \\
A_{S Q} \\
D_{S Q} \cdot P \\
A_{S Q} \cdot P\end{array}$ & $\begin{array}{l}4.4 \\
2.9 \\
8.0 \\
-\end{array}$ & $\begin{array}{l}5.3 \\
2.8 \\
6.7 \\
3.1\end{array}$ & $\begin{array}{l}6.2 \\
3.1 \\
7.7 \\
4.0\end{array}$ & $\begin{array}{l}6.2 \\
3.5 \\
6.6 \\
5.1\end{array}$ & $\begin{array}{l}7.1 \\
3.4 \\
8.0 \\
4.1\end{array}$ & $\begin{array}{l}7.1 \\
3.7 \\
7.5 \\
4.5\end{array}$ & $\begin{array}{l}7.1 \\
4.2 \\
7.3 \\
6.4\end{array}$ & $\begin{array}{r}8.9 \\
3.9 \\
10.9 \\
7.5\end{array}$ & $\begin{array}{c}8.9^{\mathrm{b}} \\
4.2 \\
10.0 \\
6.5\end{array}$ \\
\hline Joshua I & $\begin{array}{l}D_{S Q} \\
A_{S Q} \\
D_{S Q} \cdot P \\
A_{S Q} \cdot P\end{array}$ & $\begin{array}{l}4.4 \\
4.0 \\
4.4 \\
4.3\end{array}$ & $\begin{array}{l}5.3 \\
3.9 \\
5.5 \\
4.0\end{array}$ & $\begin{array}{l}6.7 \\
4.2 \\
7.5 \\
4.9\end{array}$ & $\begin{array}{l}7.6 \\
4.0 \\
8.6 \\
4.1\end{array}$ & $\begin{array}{r}8.9 \\
4.0 \\
11.5 \\
4.4\end{array}$ & $\begin{array}{l}8.9 \\
4.0 \\
9.9 \\
4.1\end{array}$ & \begin{tabular}{r|}
9.3 \\
4.1 \\
10.3 \\
4.2
\end{tabular} & $\begin{array}{r}9.8 \\
4.0 \\
10.7 \\
4.0\end{array}$ & $\begin{array}{r}10.2 \\
3.9 \\
11.2 \\
3.8\end{array}$ \\
\hline Joshua II & $\begin{array}{l}D_{S Q} \\
A_{S Q} \\
D_{S Q} \cdot P \\
A_{S Q} \cdot P\end{array}$ & $\begin{array}{r}14.7 \\
3.5 \\
14.7 \\
3.5\end{array}$ & $\begin{array}{r}18.7 \\
3.6 \\
20.0 \\
3.9\end{array}$ & $\begin{array}{r}21.3 \\
3.6 \\
23.2 \\
3.7\end{array}$ & $\begin{array}{r}24.0 \\
3.6 \\
26.6 \\
3.6\end{array}$ & $\begin{array}{c}24.9^{a} \\
3.8 \\
26.4 \\
4.1\end{array}$ & $\begin{array}{c}26.7^{\mathrm{a}} \\
3.8 \\
28.6 \\
3.9\end{array}$ & $\begin{array}{c}28.9^{a} \\
3.8 \\
31.4 \\
4.1\end{array}$ & $\begin{array}{c}29.8^{\mathrm{a}} \\
3.8 \\
31.9 \\
4.0\end{array}$ & $\begin{array}{c}32.0^{\mathrm{b}} \\
4.0 \\
34.9 \\
4.6\end{array}$ \\
\hline Piñon & $\begin{array}{l}D_{S Q} \\
A_{S Q} \\
D_{S Q} \cdot P \\
A_{S Q} \cdot P\end{array}$ & $\begin{array}{l}4.0 \\
4.5 \\
5.3 \\
9.4\end{array}$ & $\begin{array}{r}6.2 \\
4.0 \\
23.0 \\
2.5\end{array}$ & $\begin{array}{r}8.0 \\
3.9 \\
28.2 \\
2.2\end{array}$ & $\begin{array}{r}10.7 \\
3.8 \\
- \\
-\end{array}$ & $\begin{array}{r}12.0 \\
3.9 \\
55.2 \\
3.9\end{array}$ & $\begin{array}{c}13.3^{a} \\
4.0 \\
37.9 \\
4.0\end{array}$ & $\begin{array}{c}14.7^{\mathrm{b}} \\
4.4 \\
33.3 \\
-\end{array}$ & $\begin{array}{c}17.8^{\mathrm{b}} \\
4.4 \\
-\end{array}$ & $\begin{array}{c}21.3^{\mathrm{b}} \\
4.3 \\
- \\
-\end{array}$ \\
\hline
\end{tabular}


Table 6, continued

\begin{tabular}{|c|c|c|c|c|c|c|c|c|c|c|}
\hline \multirow[b]{2}{*}{ Grid } & \multirow[b]{2}{*}{ Variable } & \multicolumn{8}{|c|}{ Day } & \multirow[b]{2}{*}{10} \\
\hline & & 2 & 3 & 4 & 5 & 6 & 7 & 8 & 9 & \\
\hline \multirow[t]{4}{*}{ ALL } & $D_{S Q}$ & $7.3^{a}$ & $9.4^{b}$ & $10.9 \mathrm{~b}$ & $12.5^{b}$ & $13.3^{\mathrm{b}}$ & $14.3^{\mathrm{b}}$ & $15.0 \mathrm{~b}$ & $15.9 \mathrm{~b}$ & $17.0^{\mathrm{b}}$ \\
\hline & $A_{S Q}$ & 3.6 & 3.7 & 3.7 & 3.7 & 3.8 & 3.8 & 3.9 & 4.0 & 4.1 \\
\hline & $D_{S Q} \cdot P$ & 8.6 & 12.0 & 13.3 & 15.5 & 15.5 & 16.2 & 16.6 & 17.8 & 19.3 \\
\hline & $A S Q, P$ & 3.8 & 4.0 & 4.1 & 4.1 & 4.1 & 4.1 & 4.3 & 4.4 & 4.6 \\
\hline
\end{tabular}

Note. - Dash indicates that probability of capture could not be calculated.

a Edge effect significant at $P<0.05$.

b Edge effect significant at $P<0.01$.

animals under study is required to know the appropriate number of days of trapping that would yield a density estimate that equals the absolute density.

Inner Square. Distribution of captures indicates a positive edge effect on the outer belt of the $12 \times 12$ grid with the belts of the inner $10 \times 10$ square essentially homogeneous in their capture success. The strongest evidence for edge effect on the outer belt is the analysis of capture success for each belt with all captures combined. Total captures on the grids were 373 on Belt 1 ( 44 stations), 145 on Belt 2 (36 stations), 156 on Belt 3 (28 stations), 93 on Belt 4 (20 stations), 47 on Belt 5 (12 stations) and 17 on Belt 6 (4 stations). Captures per station on the outer belt were significantly greater $(P<.001)$ than captures per station on the inner $10 \times 10$ square. Chi-square analysis indicated that captures per station were not different between the second belt and the inner $8 \times 8$ square. The second set of evidence for positive edge effect on the outer belt only was the consideration of captures on individual grids. Edge effect was significant for 7 of the 12 grids on day 10 (Table 6). In addition, the only grids for which the edge effect was not positive were Barrel on days 2-4, Ocotillo on days 2-10 and Creosote III on days 2-4 (this is illustrated in Table 6 when the estimate of $\left.A_{\mathrm{sQ}}<3.24 \mathrm{ha}\right)$. Finally, none of the tests of the inner $8 \times 8$ square versus Belt 2 indicated a significant positive edge effect for Belt 2 . As a result of these analyses, we considered the inner $10 \times 10$ square to be homogeneous in capture success and used the estimate of numbers in this area $(2.25 \mathrm{ha})$ to estimate density.

Density estimates using the inner square $\left(D_{S Q}\right)$ were analyzed for days 2 through 10 for all species combined on each grid (Table 6). $D_{S Q}$ increased with time during the trapping period as did $D_{S M}$ although $D_{S Q}$ was usually smaller than $D_{S M}$ (Tables $4 \& 6$ ). Values of $D_{S Q}$ for 10 day totals of all species ranged from 4.0 to 36.9 animals/ha (Table 6) which was smaller than the range of $D_{S M}(3.7-51.5$ animals/ha). Day 10 values of $D_{S Q}$ for all species combined ranged from 72 to $108 \%$ of 
the corresponding $D_{S M}$ on individual grids with an average of $82 \%$ over all grids.

Estimates of area effectively trapped $\left(A_{S(2}\right)$ based on all species also Table T

Densitics in animals/ha $(D,(), D(C), P)$ and areas effectively lrapped $(A \leq 0, A s 0, p)$ of six common species $\left(N_{(j}>30\right)$ for davs 2 through 10 usme the Inter Square techmque.

\begin{tabular}{|c|c|c|c|c|c|c|c|c|c|c|}
\hline \multirow{2}{*}{$\begin{array}{l}\text { Specie. } \\
\text { Grid }\end{array}$} & \multirow[b]{2}{*}{ Variable } & \multicolumn{8}{|c|}{ Day } & \multirow[b]{2}{*}{10} \\
\hline & & 2 & 3 & 4 & 5 & 6 & 7 & 8 & 9 & \\
\hline \multicolumn{11}{|l|}{ P. formosus } \\
\hline \multirow[t]{4}{*}{ Barrel } & $D_{S O}$ & 3.9 & 12.4 & 14.7 & 16.4 & 17.8 & 19.6 & 20.0 & 31.8 & $\begin{array}{r}22.7 \\
39\end{array}$ \\
\hline & $A_{S O}$ & $2 . \overline{1}$ & 2.8 & 2.9 & 3.0 & 3.2 & 3.2 & 3.2 & 3.2 & 3.2 \\
\hline & $1)>() .1$ & 12.5 & 32.2 & 21.5 & 22.0 & 22.0 & 24.2 & 22.8 & 25.6 & 26.3 \\
\hline & $A_{()}, p$ & 3.9 & 3.9 & 3.4 & 4.1 & 4.6 & 3.9 & $3 . \overline{5}$ & 3.7 & 3.6 \\
\hline \multirow[t]{4}{*}{ Joshua II } & $D_{x(s)}$ & 3.6 & 4.4 & 4.9 & 5.3 & 5.3 & $6 . ?$ & 6.7 & 6.7 & $7.1^{\mathrm{a}}$ \\
\hline & $A \leq 0$ & 3.9 & 3.6 & 3.5 & 3.8 & 4.1 & 3.9 & 4.2 & 4.2 & 4.4 \\
\hline & $D_{\mathrm{sQ}} \cdot \mathrm{P}$ & 3.6 & 4.6 & 5.1 & 5.6 & 5.4 & 6.7 & 7.2 & 7.0 & 7.6 \\
\hline & $A_{50} \cdot P$ & 3.9 & 3.5 & 3.4 & 3.8 & 4.4 & 3.9 & 4.9 & 4.5 & 4.9 \\
\hline \multicolumn{11}{|l|}{ P. crinitus } \\
\hline \multirow{3}{*}{ Boulder } & $D_{S O}$ & $6.7^{\mathrm{a}}$ & $\begin{array}{l}8.4^{b} \\
5.0\end{array}$ & $9.8^{\mathrm{b}}$ & $\begin{array}{l}10.7 \mathrm{~b} \\
5.0\end{array}$ & $\begin{array}{c}11.1^{10} \\
5.0\end{array}$ & $\begin{array}{l}11.1^{11} \\
5.2\end{array}$ & 5.4 & $\begin{array}{c}11.6^{10} \\
57\end{array}$ & $\frac{12.9^{\circ}}{5.6}$ \\
\hline & $\begin{array}{l}\text { Aso } \\
\text { Dso.p }\end{array}$ & $\begin{array}{l}4.4 \\
-\end{array}$ & $\begin{array}{r}5.0 \\
21.9\end{array}$ & $\begin{array}{r}5.1 \\
15.1\end{array}$ & $\begin{array}{r}5.0 \\
13.7\end{array}$ & $\begin{array}{r}2.1 \\
15.1\end{array}$ & 12.2 & 11.4 & 11.9 & $\begin{array}{l}1.0 \\
13.9\end{array}$ \\
\hline & Aso.P & - & 32.6 & 6.9 & 5.1 & 4.2 & 5.3 & 5.7 & 6.3 & 6.1 \\
\hline \multirow[t]{4}{*}{ Joshua 11} & DSO & 6.2 & 6.7 & 7.6 & 8.4 & 8.4 & $3.4^{4}$ & 9.3 .1 & 10.2 & $11.6^{\mathrm{b}}$ \\
\hline & $A \subseteq O$ & 3.5 & 4.0 & 3.8 & 3.8 & 4.1 & 4.3 & 4.2 & 4.0 & 4.3 \\
\hline & DSO.P & 6.2 & 6.7 & 7.7 & 8.7 & 8.5 & 8.5 & 9.5 & 10.7 & 13.9 \\
\hline & $A_{S O} \cdot P$ & 3.6 & 4.2 & 3.9 & 3.8 & 4.4 & 4.4 & 4.4 & 4.1 & 5.2 \\
\hline \multicolumn{11}{|l|}{ P. maniculatus } \\
\hline \multirow{3}{*}{ Juniper } & Dso & 4.0 & $\begin{array}{l}5.3 \\
4.1\end{array}$ & $\begin{array}{l}6.2 \\
4.0\end{array}$ & $\begin{array}{l}67 . \\
4.0\end{array}$ & $\begin{array}{l}6.7 \\
4.2\end{array}$ & $\begin{array}{l}7.6^{\mathrm{a}} \\
4.2\end{array}$ & $\begin{array}{l}7.6^{\mathrm{a}} \\
4.2\end{array}$ & $\begin{array}{l}8.0 \\
4.1\end{array}$ & $\begin{array}{l}8.4 \\
4.1\end{array}$ \\
\hline & $\begin{array}{l}A_{S} \\
D s\end{array}$ & 4.0 & $\begin{array}{l}4.1 \\
5.8\end{array}$ & $\begin{array}{l}4.0 \\
7.0\end{array}$ & $\begin{array}{l}4.0 \\
7.2\end{array}$ & $\begin{array}{l}4.2 \\
6.9\end{array}$ & $\begin{array}{l}4.2 \\
8.2\end{array}$ & 7.8 & 8.4 & $\begin{array}{l}4.1 \\
8.9\end{array}$ \\
\hline & A SQ.P & $\overline{-}$ & 4.6 & 4.1 & 4.2 & 4.3 & 4,4 & 4.4 & 4.1 & 4.2 \\
\hline \multirow[t]{4}{*}{ Piñon } & Dso & 1.8 & 3.1 & 4.4 & 5.3 & 5.3 & 6.2 & 6.7 & $8.0^{\mathrm{a}}$ & $10.2^{8}$ \\
\hline & ASO & 4.5 & 3.5 & 3.2 & 3.4 & 3.8 & 3.5 & 3.9 & 4.2 & 4.2 \\
\hline & DSO. $P$ & 2.0 & - & - & - & 8.2 & 11.9 & 11.0 & 26.0 & - \\
\hline & $A_{S O} \cdot P$ & 6.2 & - & - & - & 5.2 & 3.3 & 7.0 & - & - \\
\hline \multirow{5}{*}{$\begin{array}{l}P \text { eremicus } \\
\text { Chaparral II }\end{array}$} & & & & & & & & & & \\
\hline & Dso & 7.6 & 8.0 & $8.0^{\mathrm{b}}$ & $8.0^{\mathrm{b}}$ & $8.0^{\mathrm{b}}$ & $8.0^{\mathrm{b}}$ & $8.4^{\mathrm{b}}$ & $3.4^{\mathrm{b}}$ & 8.96 \\
\hline & $A_{S O}$ & 4.0 & 4.1 & 4.6 & 4.9 & 5.0 & 5.1 & 5.1 & 5.1 & 5.3 \\
\hline & $D_{S O} \cdot P$ & 7.7 & 8.0 & 8.0 & 8.0 & 8.0 & 8.0 & 8.4 & 8.4 & 8.9 \\
\hline & $A S Q \cdot P$ & 4.3 & 4.3 & 4.9 & 5.0 & 5.1 & 5.2 & 5.2 & 5.2 & 5.4 \\
\hline \multirow{5}{*}{$\begin{array}{l}\text { P. truei } \\
\text { Piñon }\end{array}$} & & & & & & & & & & \\
\hline & Dso & 0.9 & 1.3 & $1.3^{\mathrm{b}}$ & $1.8^{\mathrm{a}}$ & $3.1^{\mathrm{a}}$ & $3.1^{\mathrm{b}}$ & $3.6^{\mathrm{b}}$ & $4.4^{\mathrm{b}}$ & $5.3^{b}$ \\
\hline & $A \subseteq O$ & 5.6 & 6.0 & 7.5 & 6.2 & 5.1 & 6.1 & 6.8 & 63 & 5.8 \\
\hline & DSO .1 & 0.9 & 1.7 & 1.4 & 2.4 & - & - & - & - & - \\
\hline & $A_{50} \cdot P$ & 5.9 & 11.0 & 14.9 & 4.6 & - & - & - & - & - \\
\hline \multirow{4}{*}{$\begin{array}{l}N \text {. lepida } \\
\text { Boulder }\end{array}$} & & $3.6^{\mathrm{a}}$ & 5.3 & $8.4^{\mathrm{a}}$ & $10.2^{\mathrm{a}}$ & $10.2^{\mathrm{a}}$ & $11.6^{3}$ & $11.6^{\mathrm{b}}$ & $12.4^{b}$ & $12.4^{r}$ \\
\hline & Aso & 4.8 & 4.3 & 4.4 & 4.3 & 4.3 & 4.1 & 4.3 & 4.5 & 48 \\
\hline & $D S O . P$ & - & - & - & - & 33.0 & 32.9 & 16.5 & 236 & 17.2 \\
\hline & $A S O \cdot P$ & - & - & - & - & 2.7 & 2.2 & 4.2 & 3.7 & 5.4 \\
\hline
\end{tabular}

Note-Dash indicates that probability of capture could not be calculated

a Edge effect significant at $P<0.05$

$D$ Edge effect significant at $P<0.01$ 
increased from day 2 through 10 on 7 grids although there were day to day fluctuations (Table 6). Values of $A_{S Q}$ for day 10 varied from 3.0 to 4.5 ha across all grids although Ocotillo was the only grid below the Standard Minimum area $(3.24 \mathrm{ha})$ and 10 grids had $A_{S Q}$ equal to or greater than 3.8 ha (Table 6).

Values of $D_{S \gamma}$ and $A_{S \zeta \gamma}$ for six common species are listed in Table 7. Increases in $D_{S Q}$ occurred over time for the individual species; however, $A_{S Q}$ did not demonstrate a consistent increase with time because of daily fluctuations. Differences in density were considerable for some species when estimates based on $A_{S Q}$ for all species combined and for individual species were compared (e.g., use of the $A_{S Q}$ for all species (4.5 ha) on Boulder resulted in an estimate of $15.9 \mathrm{P}$. crinitus/ha whereas use of the individual $A_{S \mathcal{V}}(5.6 \mathrm{ha})$ resulted in an estimate of $12.9 \mathrm{P}$. crinitus/ha.

Values of $D_{S Q \cdot P}$ and $A_{S(2, P}$ were calculated for days 2 through 10 for all species captured on each grid (Table 6 ) as well as for the six common species (Table 7). Examination of Table 6 suggests that $A_{S Q} \cdot 1$, leveled out ( 3 or more days with approximately the same $A_{S Q \cdot P}$ ) on only 8 of 12 grids, excluding Juniper, Creosote II and II and Piñon. Average $A_{S Q \cdot P}$ and $D_{S Q \cdot 1}$ values based on the plateau effect were 3.7 ha and 31 animals/ha for Barrel (days $7-10$ ), 5.1 and 41 for Boulder (days $8-10$ ), 3.8 and 8 for Chaparral I (days $5-10$ ), 4.3 and 20 for Chaparral iI (days 5-10), 2.9 and 4 for Ocotillo (days 6-10), 3.5 and 7 for Creosote I (days $4-7$ ), 4.2 and 10 for Joshua I (days 5-9) and 4.0 and 30 for Joshua II (days 6-9). For individual species, $A_{S Q \cdot P}$ reached a plateau for only 5 of 9 analyses (Table 7 ). Average $A_{S Q \cdot P}$ and $D_{S Q \cdot P}$ values were 3.7 ha and 25 animals/ha for $P$. formosus on Barrel (days $7-10$ ), 4.8 and 7 for $P$. formosus on Joshua II (days 8-10), 4.3 and 9 for $P$. crinitus on Joshua II (days 6-9), 4.2 and 8 for P. maniculatus on Juniper (days 4-10) and 5.1 and 8 for $P$. eremicus on Chaparral II (days $4-9$ ).

Assessment Line. Accumulative captures along the assessment lines for all species combined are summarized in Table 8 . The effects of removal of animals on the grid can be seen in the change in rate of animals captured per station along the assessment line with considerable variability in the width of this effect $(-7.5$ to $172.5 \mathrm{~m}$ in from the grid edge; Table 8). Except for the Ocotillo grid, $W_{A L}$ for all species combined agreed closely with the break in captures seen in the lists of accumulative captures (Tables $8 \& 9$ ). The range of $W_{A L}$ for all species was -19.3 to $71.3 \mathrm{~m}$ not including the Ocotillo grid which had an unreasonable $W_{A L}$ of $229.5 \mathrm{~m}$. The areas of effect $\left(A_{A L}\right)$ for all species ranged from 1.6 to 34.4 ha with eight of the 12 grids in the range of 3.2 to 9.1 ha. The proportions of animals removed $(R)$ for all species also varied considerably with a range of 0.34 to 0.91 . The area effectively 


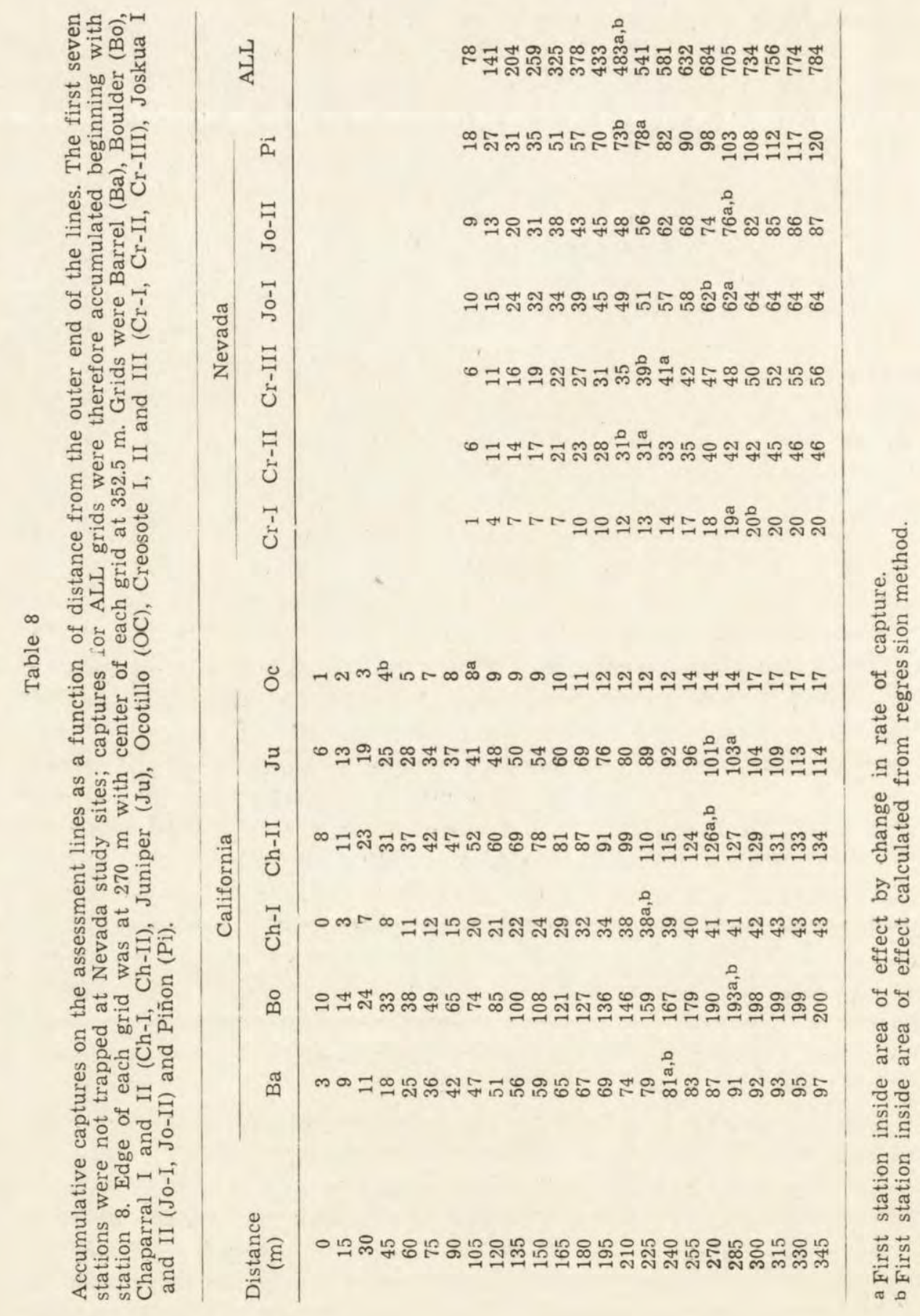


trapped (i.e., the area from which $100 \%$ removal of animals would yield $N_{G}$ and be equal to $A_{A L} \times R$ ) varied from 1.3 to 16.9 ha (average= $3.86 \mathrm{ha}$ ). Without Ocotillo the range was 1.3 to 5.1 ha (average $=2.68 \mathrm{ha}$ ). Density estimates $\left(D_{A L}\right)$ for all species were more variable, $0.7-114.4$ animals/ha, than the other density estimates (Tables 4,6 \& 9).

Area of effect, per cent animals removed and density estimates are

Table 9

Grid captures $\left(N_{G}\right)$, widths of area of effect in meters $\left(W_{A L}\right)$, areas of effect in hectares $\left(A_{A L}\right)$, proportions removed $(R)$ and density estimates in animals/ha $\left(D_{A L}\right)$ using the Assessment Line technique. Values for individual species are given only when captures on assessment lines were $N>30$ in California and $N>20$ in Nevada.

\begin{tabular}{llrrrrr}
\hline \multicolumn{1}{c}{ Grid } & \multicolumn{1}{c}{ Species } & $N_{G}$ & $W_{A L}$ & $A_{A L}$ & $R$ & $D_{A L}$ \\
\hline Barrel & All & 93 & 33.3 & 5.3 & 0.63 & 27.9 \\
& P. formosus & 73 & 35.7 & 5.5 & 0.61 & 21.8 \\
Boulder & All & 190 & -7.2 & 2.3 & 0.86 & 96.1 \\
& $P$. crinitus & 79 & 1.6 & 2.8 & 0.90 & 31.3 \\
Chaparral 1 & N. lepida & 68 & 32.7 & 5.2 & 0.52 & 25.1 \\
& All & 32 & 50.0 & 6.8 & 0.75 & 6.3 \\
Chaparral II & $P$. eremicus & 20 & 50.4 & 6.9 & 0.68 & 4.3 \\
& All & 88 & 7.4 & 3.2 & 0.74 & 37.2 \\
Juniper & P. eremicus & 47 & -8.4 & 2.2 & 0.82 & 26.1 \\
& All & 81 & 9.6 & 3.4 & 0.41 & 58.1 \\
Ocotillo & $P$. maniculatus & 35 & 49.0 & 6.7 & 0.80 & 6.5 \\
Creosote I & D. merriami & 16 & -68.8 & 0.1 & 0.55 & 290.9 \\
Creosote II & All & 12 & 229.5 & 34.4 & 0.49 & 0.7 \\
Creosote III & All & 33 & -19.3 & 1.6 & 0.86 & 24.0 \\
Joshua I & All & 28 & 71.3 & 9.1 & 0.42 & 7.3 \\
& All & 37 & 46.4 & 6.5 & 0.45 & 12.6 \\
& All & 40 & 10.4 & 3.4 & 0.91 & 12.9 \\
Joshua II & $P$. formosus & 17 & 27.2 & 4.8 & 0.84 & 4.2 \\
& D. merriami & $\gamma$ & 1.6 & 2.8 & 1.00 & 2.9 \\
Piñon & All & 151 & -5.6 & 2.4 & 0.55 & 114.4 \\
& P. crinitus & 61 & 36.0 & 5.5 & 0.67 & 16.6 \\
& All & 91 & 62.8 & 8.1 & 0.34 & 33.0 \\
& $P$. maniculatus & 43 & 69.1 & 8.8 & 0.29 & 16.8 \\
& P. truei & 31 & -13.3 & 1.9 & 0.34 & 48.0 \\
\hline
\end{tabular}

also summarized in Table 9 for common species caught on the assessment lines ( $N>30$ for California sites, $N>20$ for Nevada sites).

An attempt was made to place confidence intervals around $R$ using the standard errors of the mean number of captures per station inside and outside of the area as outlined by S mith et al. (1975) but the confidence intervals were unreasonably large. For example, confidence limits for $R$ on Creosote I were $0.00-1.00$, suggesting that these captures represented zero to $100 \%$ of the rodents in the area of the grid. 
Probabilities of capture on the assessment lines inside the area of effect $\left(P_{c \cdot I n}\right)$ were calculated for only seven of the 12 grids whereas values of $P_{\text {c.Out }}$ could be calculated for nine of 12 grids. As a result, both values were available for only five grids (Table 10). When $P_{c}$ values were not estimated it was because most captures occurred late in the four day period so that $P_{c}$ values were not calculable from tables in J a nion et al. (1968). The failure to estimate corrected proportion of removal $\left(R_{p}\right)$ for the majority of grids argues against the overall effectiveness of the Assessment Line technique.

To examine the generality of the Assessment Line method, captures on assessment lines from all grids were combined beginning with

\section{Table 10}

Probabilities of capture inside $\left(P_{c}, I_{n}\right)$ and outside $\left(P_{c}\right.$, Out $)$ of the area of effect, proportion removed corrected for differences in probability of capture $\left(R_{P}\right)$ and density in animals/ha $\left(D_{A L} \cdot P\right)$ using the Assessment Line technique.

\begin{tabular}{|c|c|c|c|c|}
\hline Grid & $P_{c} \cdot I n$ & $P_{c}$. Out & $R_{P}$ & $D_{A L} \cdot P$ \\
\hline Barrel & 0.28 & - & - & - \\
\hline Boulder & 0.08 & 0.25 & 0.65 & 127.1 \\
\hline Chaparral I & 0.46 & 0.23 & 0.82 & 5.7 \\
\hline Chaparral II & - & 0.29 & - & - \\
\hline Juniper & 0.20 & 0.25 & 0.31 & 76.9 \\
\hline Ocotillo & - & - & - & - \\
\hline Creosote I & 0.34 & - & - & - \\
\hline Creosote II & 0.31 & 0.36 & 0.38 & 8.1 \\
\hline Creosote III & - & 0.08 & - & - \\
\hline Joshua I & 0.40 & 0.34 & 0.92 & 12.9 \\
\hline Joshua II & - & 0.29 & - & - \\
\hline Piñon & - & 0.14 & - & - \\
\hline
\end{tabular}

Note. - Dash indicates that probability of capture could not be calculated.

Station 8 (Stations $1-7$ were not trapped in Nevada). The combined data indicated two areas of partial removal (Table 8). The area of no effect extended from station 8 to 14 with a rate of capture of 3.95 animals $/ \mathrm{m}$ (slope of the regression equation). The outer area of partial removal extended from station 15 to 19 (corresponds to the outer row on the grid) with a capture rate of 3.29 animals $/ \mathrm{m}$ whereas the inner area of partial removal extended from station 20 to the center of the grid with a capture rate of 1.32 animals $/ \mathrm{m}$. Using the regression method, the width of the area of effect $\left(W_{A L}\right)$ was calculated as $76.9 \mathrm{~m}$ whereas the edge of effect for the inner area of partial removal was $-4.3 \mathrm{~m}$. From these values, the area of effect was $9.66 \mathrm{ha}$, the inner area of partial removal was 2.45 ha and, from the difference, the outer 
area of partial removal was 7.21 ha. From the shlopes of the regression lines, the proportion of animals removed from the outer area was 0.167 and from the inner area 0.666 . These values were then used to correct both areas of partial removal to areas effectively trapped, with an outer area of 1.20 ha, an inner area of 1.63 ha and a total area effectively trapped of 2.83 ha. The average number of animals caught per grid was 73.0 and, therefore, the average density per grid was 25.8 anımals/ha. This density estimate was considerably lower than the average of individual $D_{A L}$ estimates of 35.9 animals/ha. In addition, the average area effectively trapped $\left(A_{A L} \times R\right.$ in Table 9$)$ was 3.86 ha which was $36 \%$ larger than the equivalent area calculated from all grids combined. With the exceedingly high estimate of the Ocotillo equivalent area (1.69 ha) removed, the average was only 2.68 ha for 11 grids.

For all grids combined, $P_{c}$ was 0.210 for the area of no removal and 0.220 for the outer area of partial removal but was not calculable for the inner area of partial removal. The proportion removed in the outer area based on the $P_{c}$ values was 0.194 and was similar to the $R$ value of 0.167 . However, the average density for all grids combined was not estimated since the $P_{c}$ value for the inner area was not calculable.

\section{DISCUSSION}

The three techniques examined gave final density estimates that were quite different with $D_{S Q}$ and $D_{A L}$ averaging 82 and $140 \%$ of $D_{S: I I}$, respectively. Estimates of $D_{S Q}$, although less than $D_{S M}$ values for day 10 , were farily consistent with a range of 72 to $108 \%$ of the corresponding $D_{S M}$ values across the 12 grids. Only one $D_{S Q}$ was greater than its respective $D_{S M}$. The $D_{A L}$ values were quite variable and ranged from 19 to $287 \%$ of $D_{S M}$. Four $D_{A L}$ estimates were less than their respective $D_{S M}$ estimates. Even with this considerable variability among the three estimators, the estimates must be relative measures of the absolute density in the area of the trap site within any of the three groups The suggestion that all three methods are measuring relative densities, regardless of whether any of the three actually is a good measure of absolute density, is borne out by the fact that all three measures group the same sites into four different density classes (low, low intermediate, high intermediate and high density areas). The low density group included only the Ocotillo site with 3.7 rodents/ha for $D_{S M}, 4.0$ for $D_{S Q}$ and 0.7 for $D_{A L}$. The low intermediate density sites included Chaparral I, Creosote I, II and III and Joshua I with a range of densities of 8.6 to 12.3 rodents/ha for $D_{S M}, 6.7$ to 10.2 for $D_{S Q}$ and 6.3 to 24.0 for $D_{A L}$. The four high intermediate sites were Chaparral II, Juniper, Piñon and 
Barrel with a range of densities of 25.0 to 28.7 rodents/ha for $D_{S M}$, 19.6 to 27.6 for $D_{S Q}$ and 27.9 to 58.1 for $D_{A L}$. The high density sites were Joshua II and Boulder with densities of 39.8 and 51.5 rodents/ha for $D_{S M}, 32.0$ and 36.9 for $D_{S Q}$ and 114.4 and 96.1 for $D_{A L}$. Finally, the three estimators were highly correlated and can be interrelated by the following simple linear regression equations: $D_{S Q}=1.0447+0.7452 D_{S M}$, $r=0.99 ; \quad D_{A L}=-12.0191+2.2415 D_{S M}, \quad r=0.89 \quad$ and $\quad D_{A L}=-13.1067+$ $2.8869 D_{S Q}, r=0.87$.

Even though all three methods may be useful for relative density comparisons, the question remains, which technique is best for estimation of absolute density of small mammals? The advantage appears to be with the Assessment Line technique in which both numbers and area are estimated as compared to the Standard Minimum and Inner Square in which numbers are estimated but area is assumed to be a constant, either the entire grid or some inner square (see review by $\mathrm{Smith}$ et al., 1975). However, does the use of the Assessment Line technique under fieid conditions meet with theoretical expectations, i.e., when captures along the assessment lines are accumulated is there an obvious break in the rate of accumulation at the edge of the area of effect? When individual grids were examined (Table 8 ), we did not observe uniform increases in numbers with distance or sharp breaks in the slopes at the edges of the area affected by the census trapping for all grids. Therefore, it would appear that individual grids do not always meet the expectations of the technique. Habitat and density heterogeneity within a single habitat type apparently result in a jagged accumulative removal line even when eight assessment lines are used on each grid (Table 8). These removal curves suggest that more than eight assessment lines are necessary to obtain a good estimate of the area affected by the grid trapping. Since it is not possible to use more than eight assessment lines with a square grid of the general size that we employed, analysis of two or more square grids or a larger rectangular grid would usually be needed to obtain reasonable density estimates with the Assessment Line method.

To assess the potential of combining data from two or more grids. we combined captures on all study sites (Table 8 ) for comparison to the theoretical expectations of the Assessment Line method. The results of this indicated an obvious area of effect which in fact consisted of two regions based on the proportion of rodents removed (see Restits). Accumulating and averaging across many assessment lines overcomes the variability in rodent dispersion due to habitat heterogeneity that prevents a clear picture from emerging when only one grid is examined, i.e., the change in slope for any one set of assessment lines is the re- 
sult of patterns of removal superimposed on patterns of dispersion, and only when many lines are pooled so that the pattern of dispersion averages into a straight line can the break due to removal be detected. Even at high densities, the use of only eight assessment lines may not overcome the problem of dispersion since none of the individual grids demonstrated the large area of effect shown by combining data from all of the grids (Table $8 \& 9$ ). This analysis suggests that when sufficient numbers of grids are studied and assessment line captures are combined across several grids, the technique meets the theoretical expectations and provides reasonable density estimates.

The rationale behind the Assessment Line method includes three points: (1) a census grid or line will remove animals from some area, (2) this area of effect can be delimited by examining captures on assessment lines set across the area subsequent to census trapping and (3) the proportion of animals removed from the area by the census grid can be estimated from differences in capture rate along the assessment lines inside and outside of the area of effect (Gentry, S mith \& Chelton, 1971; Kaufman et al., 1971; Smith et al., 1971, 1975; and above). After consideration of the large outer area of partial removal with its corresponding low value of $R$ when all grids were combined, its seems appropriate to modify the rationale of the method to state that we are measuring the area in which animals are affected but not necessarily removed. Census trapping causes the formation of an area of removal (partial and/or complete depending on length of trapping period); however, animals outside of this area may move into the void prior to assessment line trapping. Movement into the partial (or complete) void causes the area of effect to be larger than the area of removal so that $W_{A L}$ is not a measure of the distance over which removal occurred, but is rather a measure of the distance over which the grid affected the rodent community during the 14 day period (10 days on the grid plus 4 days before completion of assessment line trapping). Calculation of density is not affected by this movement since the proportion of animals removed from the affected area is still being estimated even though the original distribution of animals has been rearranged. To summarize, we can say that even though the area of effect has been discussed as an area from which animals were removed, this cannot be confirmed and should therefore be thought of as an area in which census trapping has caused both removal and rearrangement of animals.

$\mathrm{S} \mathrm{mith}$ et al. (1971) outlined three general criticisms of the Assessment Line method: (1) mortality during long trapping periods, (2) failure to work at low densities and (3) differences in probability of capture 
on the assessment lines inside and outside of the area of effect. The ten day trapping period for this study was based on the expectation that a significant proportion of animals would be captured from the area of each grid without excessive mortality that would alter density estimation. We assume that both goals were accomplished. Low densities are a problem for any density estimation technique; however, for the Assessment Line method, the minimum estimable density is determined by the distribution of the animals on the study site. We have already indicated that for most grids, the interaction of rodent density and dispersion would require the use of more than eight assessment lines and that pooling the data from several sets of assessment lines would be needed to calculate reasonable estimates of density. Using the approach of Janion et al. (1968), we attempted to test for differences in $P_{c}$ inside and outside of the area of effect; however, estimation of a $P_{c}$ value was very inconsistent (only 16 of 24 values were calculable). Calculation of an average probability of capture requires that the population at risk remains constant over time. Furthermore, this technique assumes a specific geometric distribution for captures through time. We know that either one or both of these expectations were definitely not met on one-third of the tests (of the remaining 16 we have no way of knowing how many are reasonable estimates of $P_{c}$ ) which suggest that the estimation of $P_{c} \cdot$ In and $P_{c}$. out does not have general applicability. The Assessment Line technique assumes that the rates of capture over distance (slope of regression equations) inside and outside of the area of effect are relative estimates of the respective densities at that point in time. Therefore, probabilities of capture need be neither constant or equal in the two areas provided the same proportion of the true densities in each area are captured along the assessment lines at the end of four days.

The Standard Minimum technique has value as an estimator of relative aensity but offers little potential for absolute density determination. This failure lies in the assumption that the grid removes animals from some constant area. However, our Assessment Line data (Table 9) show that the area affected is not constant across grids. Estimation of numbers from Hayne (1949) or Janion et al. (1968) does not alter the problem of absolute density estimation. The use of the Standard Minimum for determination of absolute density necessitates knowledge of the day on which the estimate of numbers is equivalent to the number in the Standard Minimum area ( 3.24 ha). If a particular rodent is studied in enough detail, it might be possible to decide upon a time period that would produce a reasonable absolute density estimate ( $\mathrm{G}$ r o- 
$\mathrm{dzinski}$ et al., 1966). However, for this study there is no way of knowing the appropriate day of trapping to use for different species. In fact, using the density value from all grids combined for Assessment Line data as the estimate, several to many more days of trapping would be required to calculate density with the Standard Minimum method.

The Inner Square technique i: basically a modification of the approach and assumptions of the Stundard Minimum technique, i.e., the densily of a constant area can be estimated by trapping for a given period of time. The difference is that the Inner Square method excludes the area and captures on the outher belt(s) from the density calculations to avoid the complications of edge effect ( $\mathrm{Hansson}, 1969$; Pelikan, 1970). However, density estimation from values of $D_{S Q}$ suffers the same problems as discussed with $D_{S M}$ and $D_{S M} \cdot p$, since with the continual increase in the value of the estimator there is no way of knowing the trapping day on which $D_{S Q}$ best approximates the true density. To overcome this problem, Hansson (1969) calculated density and area for individual species $\left(D_{S Q}, P\right.$ and $\left.A_{S Q}, P\right)$ from the probabilities of capture for each species in the inner square and entire grid. He then chose as the best estimate of density the value for the days when the area effectively trapped by the grid reached a plateau. The plateau theoretically occurs only during the time when animals whose home ranges overlap the grid are caught. Increase in the areal estimate following the plateau is due to the collapse of animals onto the grid whose home ranges never overlapped with the grid. However, application of this plateau effect was less than satisfying since only 5 of 9 individual species and 8 of 12 all-species estimates were calculable. Most of these $D_{S Q}$. P values were also considerably different from the values of $D_{A L}$ (Tables 6, 7 \& 9).

$\mathrm{Bar}$ behenn (1974) examined the use of the Inner Square procedures for individual small mammal species using an $8 \times 8$ grid. From his anglyses, Barbehenn concluded that calculation of $D_{S Q} \cdot p$ from the first four days of trapping was successful and indicated that the technique should have general applicability. Variability in $D_{S Q} . P$ for individual species during the trapping period in our study (Table 7) is enough to question the generality of this method. This variability in density is due to variability in the probability of capture on the inner square (the range of variability of $P_{\mathrm{c}} \cdot I_{S}$ was similar to the range of $P_{c}$ illustrated in Table 5). The weakness in the overall technique is probably related to the fact that differences in behavioral response to traps and movements among different subgroups even within a single species negate the assumptions for the calculation of an average pro- 
bability of capture. Estimation of $D_{S Q}, p$ for all species may be even more questionable since we calculated an average probability of capture across several species which may have had different probabilities of capture. Finally, $D_{S Q}, p$ for all species combined over all grids was only 50 to $80 \%$ of the $D_{A L}$ value for all grids and $D_{A L}$ for all grids is the best estimate of average density (based on the fit of the data to the theoretical model). Based on these considerations, we argue that although the Inner Square technique may work for certain species under certain conditions, it does not have general applicability.

Acknowledgements: We thank S. Kaufman, S. Presley, D. Roe, M. W. Smith and W. B. Wray for assistance in the field.

\section{REFERENCES}

1. A damczyk K. \& Ryszkowskj L., 1968: Estimation of the density of a rodent population using stained bait. Acta theriol., 13: 295-311.

2. A u la k W., 1967: Estimation of small mammal density in three forest bictopes Ekologia Polska, Ser. A, 15: 755-778.

3. Barbehenn K. R., 1974: Estimating density and home range size with removal grids: the rodents and shrews of Guam. Acta theriol., 19: 191-234.

4. Buchalc $\mathrm{c} y \mathrm{k}$ T. \& Pucek Z.. 1968: Estimation of the numbers of Microtus oeconomus using the Standard Minimum method. Acta theriol., 13: 461-482.

5. Gentry J. B., Smith M. H. \& Chelton J. G., 1971: An evaluation of the octagon census method for estimating small mamnal populations. Acta theriol.. 16: $149-159$.

6. Grod\%inski W., Pucek Z. \& Ryszkowski L., 1966: Estimation of rodent numbers by means of prebaiting and intensive removal. Acta theriol. 11: $297-314$

7. Hans on L., 1969: Home range, population structure and density estimates at removal catches with edge effect. Acta theriol., 14: 153-160.

8. Hay ne D. W., 1949: Two methods of estimating population from trapping records. J. Mammal., 30: 399-411.

9. Janion S. M., Rys\%kowski L. \& Wierzbowska T., 1968: Estimation of number of rodents with variable probability of capture. Acta theriol., 13. $285-294$

10. Kaufman D. W., Smith G. C., Jones R. M., Gentry J. B. \& $\mathrm{Smith}$ M. H., 1971: Use of assessment lines to estimate density of small mammals. Acta theriol., 16: 127-147.

11. Pelikán J., 1970: Testing and elimination of the edge effect in trapping small mammals. [In: »Energy flow through small mammal populations $\alpha$, Eds. Petrusewicz K. \& Ryszkowski L.]. Polish Sci. Publ.: 57-61. Warszawa.

12. Smith M. H., Bless ing R., Chelton J. G., Gentry J. B., Golley F. B. \& M c Ginnis J. T., 1971: Determining density for small mammal populations using a grid and assessment lines. Acta theriol., 16: 105-125. 
13. Smith M. H., Gardner R. H., Gentry J. B., Kaufman D. W. \& O'F a rrell M. J., 1975: Density estimation of small mammal populations. [In: "Small mammals: their productivity and population dynamics«, Eds. Golley F. B., Petrusewicz K. \& Ryszkowski L.]. International Biol. Programme, 5: 25-53.

Accepted, November 5, 1977.

Donald W. KAUFMAN, John B. GENTRY, Glennis A. KAUFMAN, Michael H. SMITH \& James G. WIENER

\section{SZACOWANIE ZAGESZCZENIA DROBNYCH SSAKOW: POROWNANIE TECHNIK STOSUJĄCYCH WYŁOW}

\section{Streszczenie}

Do przetestowania względnej efektywności szacowania zagęszczenia drobnych ssaków metodami Standard Minimum, Wewnętrznego Kwadratu i Linii Oceniających (Assessment Lines) prowadzono odłów $\mathrm{z}$ usuwaniem na 12 powierzchniach usytuowanych $\mathrm{w}$ różnych środowiskach. Na każdej powierzchni, z siecią pułapek ustawionych w więźbie $12 \times 12$ prowadzono przez 10 dni wyłów, następnie przenoszono pułapki na linie oceniające, na których wyłów trwał 4 dni. Dzięki zróżnicowaniu środowisk skład ilościowy i gatunkowy zwierząt był różny a zakres zmienności tych parametrów bardzo szeroki bo obejmujący 12-167 gryzoni/powierzchnię i 2-10 gatunków/pow. (Tabele 1-3). Zagęszczenie szacowane metodą Standard Minimum wahało się od 3.7 do 51.5 osobnika/ha (Tabele 4 i 5 ). Zakres zmienności tego estymatora oszacowany metodą Wewnętrznego Kwadratu wynosi $4.0-36.9$ zwierząt/ha (Tabele 6 i 7) a metodą Linii Oceniających odpowiednio 0.7-114.4 (Tabele 8 i 9). Wszystkie stosowane metody szacunku nadają się do porównań zagęszczenia względnego, ale ich przydatność dla szacowania absolutnego zagęszczenia jest ograniczona. W tym przypadku najlepsza jest metoda Linii Oceniających, z zastrzeżeniem, że najczęściej nie wystarcıy użycie ośmiu linii oceniających. W dyskusji szczególowo potraktowano problemy związane ze stosowaniem wszystkich porównywanych metod szacowania zagęszczenia. 